\title{
$\aleph$-injective Banach spaces and $\aleph$-projective compacta
}

\author{
Antonio Avilés, Félix Cabello Sánchez, Jesús M. F. Castillo, \\ Manuel González and Yolanda Moreno
}

\begin{abstract}
A Banach space $E$ is said to be injective if for every Banach space $X$ and every subspace $Y$ of $X$ every operator $t: Y \rightarrow E$ has an extension $T: X \rightarrow E$. We say that $E$ is $\aleph$-injective (respectively, universally $\aleph$-injective) if the preceding condition holds for Banach spaces $X$ (respectively $Y$ ) with density less than a given uncountable cardinal $\aleph$. We perform a study of $\aleph$-injective and universally $\aleph$-injective Banach spaces which extends the basic case where $\aleph=\aleph_{1}$ is the first uncountable cardinal. When dealing with the corresponding "isometric" properties we arrive to our main examples: ultraproducts and spaces of type $C(K)$. We prove that ultraproducts built on countably incomplete $\aleph$-good ultrafilters are $(1, \aleph)$-injective as long as they are Lindenstrauss spaces. We characterize $(1, \aleph)$-injective $C(K)$ spaces as those in which the compact $K$ is an $F_{\aleph-}$ space (disjoint open subsets which are the union of less than $\aleph$ many closed sets have disjoint closures) and we uncover some projectiveness properties

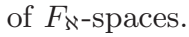

\section{Introduction}

A Banach space $E$ is said to be injective if for every Banach space $X$ and every subspace $Y$ of $X$, each operator $t: Y \rightarrow E$ admits an extension $T: X \rightarrow E$. In this paper we consider two weak forms of injectivity that arise by limiting the size of either the subspace $Y$ or the containing space $X$ in the preceding definition. Let us label them right now. Recall that the density character dens $(X)$ of a topological space $X$ is the smallest cardinal a dense subset of $X$ can have.

Definition 1.1. Let $E$ be a Banach space, $\aleph$ an uncountable cardinal and $\lambda \geq 1$ a real number. We say that $E$ is $\aleph$-injective if for every Banach space $X$ with $\operatorname{dens}(X)<\aleph$ and each subspace $Y \subset X$, every operator $t: Y \rightarrow E$ can be extended

Mathematics Subject Classification (2010): Primary 46B03, 54B30, 46B08; Secondary 54C15, 46B26.

Keywords: Injective Banach spaces, cardinality assumptions, projective compacta. 
to an operator $T: X \rightarrow E$. We say that $E$ is $(\lambda, \aleph)$-injective if we can always find some extension $T$ such that $\|T\| \leq \lambda\|t\|$. Replacing the condition $\operatorname{dens}(X)<\aleph$ by dens $(Y)<\aleph$ one obtains the definitions of universally $\aleph$-injective and universally $(\lambda, \aleph)$-injective spaces.

The choice $\aleph=\aleph_{0}$ (the first infinite cardinal) would not be too interesting to us: all Banach spaces would be "universally $\aleph_{0}$-injective". Asking for an uniform bound on the norm of the extension, that is, considering "(universally) $\left(\lambda, \aleph_{0}\right)$ injective" spaces one arrives to the class of $\mathscr{L}_{\infty}$-spaces which has been widely studied in Banach space theory in connection with the extension of compact operators; see Section 4 of [39]. Moving to the first uncountable cardinal $\aleph_{1}$ one obtains the classes of separably injective and universally separably injective Banach spaces, which also attracted attention. (Admittedly, that the resulting name for separably injective spaces turns out to be " $\aleph_{1}$-injective" is perhaps surprising. Nevertheless, we have followed the uses of set theory were properties labeled by a cardinal $\aleph$ always indicate that something happens for sets whose cardinality is strictly less than $\aleph$.) It is worth noticing that Zippin proved in the late seventies that every infinite dimensional separable and separably injective Banach space has to be isomorphic to $c_{0}$, the space of all null sequences with the sup norm, and so even in the case $\aleph=\aleph_{1}$ one is mainly concerned with nonseparable Banach spaces. We refer the reader to [39], [4] for an account and further references.

Let us describe the plan of the paper and highlight its main results. Section 2 is preliminary; it contains some definitions together with the minimal background on exact sequences of Banach spaces one needs to read the paper. In Section 3 we extend a variety of results in [4] about (universal) separably injective Banach spaces to higher cardinals. However, we found no reasonable generalization for a considerable portion of the results proved in [4] for $\aleph_{1}$ and so the resulting picture is rather incomplete. In contrast to Section 3, which deals mainly with "isomorphic" properties, the ensuing Section 4 is of "isometric nature" and studies some special properties of $(1, \aleph)$-injective spaces and their universal relatives. We use ideas of Lindenstrauss to give a characterization of $(1, \aleph)$-injective spaces by means of intersection properties of balls (Lemma 4.1) and we prove that under the generalized continuum hypothesis $(1, \aleph)$-injective spaces are in fact universally $(1, \aleph)$-injective. The characterization just mentioned opens the door to the main examples worked in Sections 5 and 6: spaces of continuous functions on compacta and ultraproducts. Theorem 5.2 unifies and extends several characterizations of $(1, \aleph)$-injective $C(K)$ spaces in terms of properties of the compact space $K$. The space $\ell_{\infty} / c_{0}=C\left(\mathbb{N}^{*}\right)$ is shown to be $\left(1, \aleph_{1}\right)$-injective but not $\left(1, \aleph_{2}\right)$-injective. Theorem 6.2 establishes that ultraproducts via $\aleph$-good ultrafilters become $(1, \aleph)$-injective whenever they are Lindenstrauss spaces. As a corollary we solve a question of Bankston by showing that ultracoproducts of arbitrary compact spaces over $\aleph$-good ultrafilters are $F_{\aleph}$-spaces. The characterization of $(1, \aleph)$-injective $C(K)$ spaces as those in which the compact $K$ is an $F_{\aleph}$-space will lead us to study projectiveness properties of these compacta which are interesting in its own right. As it is well-known, a Banach space is 1-injective if and only if it is isometrically isomorphic to $C(K)$ for some extremely disconnected compact space $K$. On the other hand, such compacta 
are precisely the projective elements in the category of compacta and continuous maps, a classical result by Gleason. This means that if $\sigma: L \rightarrow M$ is a continuous surjection then any continuous map $\varphi: K \rightarrow M$ lifts to $L$ in the sense that there is $\tilde{\varphi}: K \rightarrow L$ such that $\varphi=\sigma \circ \tilde{\varphi}$. Motivated by these correspondences, in Section 7 we explore the projectiveness properties of compact $F_{\aleph}$-spaces since, as mentioned before, a compact space $K$ is an $F_{\aleph}$-space precisely when the Banach space $C(K)$ is $(1, \aleph)$-injective. According to a result of Neville and Lloyd, totally disconnected $F_{\aleph}$-spaces can be characterized as those compact spaces which are projective with respect to surjections $\sigma: L \rightarrow M$ between compacta of weight less than $\aleph$. Theorem 7.4 states that this is also equivalent to projectiveness with respect to compacta that are hereditarily of Lindelöf number below $\aleph$. At the end of Section 7 we present a characterization of $F_{\aleph}$-spaces without any connectedness hypothesis, namely, that a compact space is an $F_{\aleph}$-space if and only if it is "projective" with respect to all affine surjections between compact convex sets of weight less that $\aleph$.

We close with a few open problems that arise naturally from the content of the paper and we were unable to resolve.

\section{Preliminaries}

\subsection{Notations, conventions}

All Banach spaces will be assumed to be real. All the results in this paper can be translated to the complex case, sometimes with some extra effort, but we have preferred not to do that.

Our notation is fairly standard, as in [27], except perhaps in that given a cardinal number $\aleph$ we denote by $\ell_{\infty}(\aleph)$ the space of all bounded functions defined on an unspecified set $\Gamma$ with $|\Gamma|=\aleph$, endowed with the sup norm and $c_{0}(\aleph)$ the closed subspace spanned by the characteristic functions of the singletons of $\Gamma$. By $\ell_{1}(\aleph)$ we denote the space of absolutely summable families of scalars indexed by $\Gamma$ with the sum norm. A Banach space $X$ is said to be a $\mathscr{L}_{\infty, \lambda}$-space if every finite dimensional subspace $F$ of $X$ is contained in another finite dimensional subspace of $X$ whose Banach-Mazur distance to the corresponding $\ell_{\infty}^{n}$ is at most $\lambda$. A $\mathscr{L}_{\infty^{-}}$

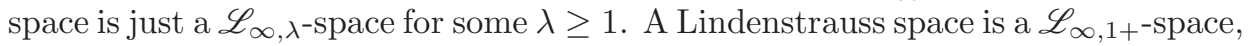
that is a Banach space which is a $\mathscr{L}_{\infty, \lambda}$-space for all $\lambda>1$.

As usual, given a compact space $K$ we denote by $C(K)$ the Banach space of all real-valued continuous functions on $K$, with the sup norm. In this paper all topological spaces are assumed to be Hausdorff. An $\mathcal{M}$-space is a Banach lattice where $\|x+y\|=\max (\|x\|,\|y\|)$ provided $x, y$ are disjoint. Each $\mathcal{M}$-space can be represented as a sublattice of some $C(K)$-space.

Throughout the paper, ZFC denotes the usual setting of set theory with the axiom of choice, while $\mathrm{CH}$ denotes the continuum hypothesis $\left(\aleph_{1}=2^{\aleph_{0}}=\mathfrak{c}\right)$ and $\mathrm{GCH}$ denotes the generalized continuum hypothesis (namely that $\aleph^{+}=2^{\aleph}$ holds for all infinite cardinals $\aleph$ ). 


\subsection{Exact sequences}

A short exact sequence of Banach spaces is a diagram

$$
0 \longrightarrow Y \stackrel{\imath}{\longrightarrow} X \stackrel{\pi}{\longrightarrow} Z \longrightarrow 0
$$

where $Y, X$ and $Z$ are Banach spaces and the arrows are operators in such a way that the kernel of each arrow coincides with the image of the preceding one. By the open mapping theorem $\imath$ embeds $Y$ as a closed subspace of $X$ and $Z$ is isomorphic to the quotient $X / \imath(Y)$.

The sequence (2.1) is said to be trivial, or to split, if there is an operator $p: X \rightarrow Y$ such that $p \imath=\mathbf{1}_{Y}$ (i.e., $\imath(Y)$ is complemented in $X$ ); equivalently, there is an operator $s: Z \rightarrow X$ such that $\pi s=\mathbf{1}_{Z}$. When properly classified and organized, the set of all possible exact sequences of the form (2.1) become a linear space, denoted by $\operatorname{Ext}(Z, Y)$, whose zero is the class of trivial sequences; see [8], [9] for explanations. For this reason one often writes $\operatorname{Ext}(Z, Y)=0$ to indicate that every sequence of the form (2.1) is trivial.

A property $\mathscr{P}$ is said to be a 3 -space property if $X$ has $\mathscr{P}$ whenever there is an exact sequence of the form (2.1) in which both $Y$ and $Z$ have $\mathscr{P}$.

\subsection{The push-out and pull-back constructions}

A thorough description of the pull-back and push-out constructions in Banach spaces can be seen in [4], [3], [9]. Everything we need to know for this paper is that given an exact sequence (2.1) and an operator $t: Y \rightarrow B$ there is a commutative diagram

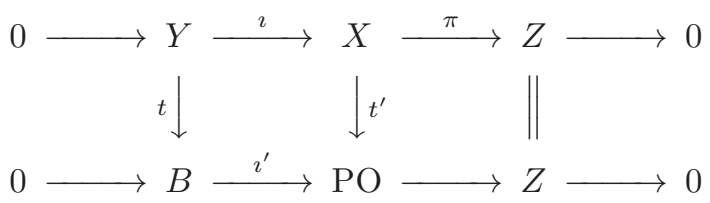

called the associated push-out diagram, in which the lower row is an exact sequence which splits if and only if $t$ extends to $X$, that is, there is an operator $T: X \rightarrow B$ such that $T \imath=t$.

Proceeding dually one obtains the associated pull-back sequence. Given an exact sequence (2.1) and an operator $u: A \rightarrow Z$ there is a commutative diagram

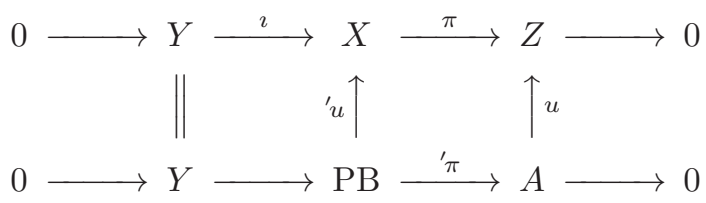

whose lower sequence is exact, and which shall be referred to as a pull-back diagram. The splitting criterion is now as follows: the lower sequence splits if and only if $u$ lifts to $X$, that is, there is an operator $U: A \rightarrow X$ such that $\pi U=u$. 


\subsection{Filters}

Recall that a family $\mathscr{F}$ of subsets of a given set $I$ is said to be a filter if it is closed under finite intersection, does not contain the empty set and one has $A \in \mathscr{F}$ provided $B \subset A$ and $A \in \mathscr{F}$. An ultrafilter on $I$ is a filter which is maximal with respect to inclusion. If $X$ is a (Hausdorff) topological space, $f: I \rightarrow X$ is a function, and $x \in X$, one says that $f(i)$ converges to $x$ along $\mathscr{F}$ (written $x=\lim _{\mathscr{F}} f(i)$ to short) if whenever $V$ is a neighborhood of $x$ in $X$ the set $f^{-1}(V)=$ $\{i \in I: f(i) \in V\}$ belongs to $\mathscr{F}$. The obvious compactness argument shows that if $X$ is compact, and $\mathscr{F}$ is an ultrafilter on $I$, then for every function $f: I \rightarrow X$ there is a unique $x \in X$ such that $x=\lim _{\mathscr{F}} f(i)$.

\subsection{The set-theoretic ultraproduct construction}

It will be used in Section 6. Let us recall some definitions, and fix notations.

Let $\left(S_{i}\right)_{i \in I}$ be a family of sets indexed by $I$ and let $\mathscr{U}$ be an ultrafilter on $I$. The set-theoretic (or model-theoretic) ultraproduct $\left\langle S_{i}\right\rangle_{\mathscr{U}}$ is the product set $\prod_{i} S_{i}$ factored by the equivalence $\left(s_{i}\right)=\left(t_{i}\right) \Leftrightarrow\left\{i \in I: s_{i}=t_{i}\right\} \in \mathscr{U}$. The class of $\left(s_{i}\right)$ in $\left\langle S_{i}\right\rangle_{\mathscr{U}}$ is denoted $\left\langle\left(s_{i}\right)\right\rangle_{\mathscr{U}}$. If we are given functions $f_{i}: S_{i} \rightarrow K$, where $K$ is some compact space, we can define another function $f:\left\langle S_{i}\right\rangle_{\mathscr{U}} \rightarrow K$ by $f\left(\left\langle s_{i}\right\rangle_{\mathscr{U}}\right)=$ $\lim _{\mathscr{U}(i)} f_{i}\left(s_{i}\right)$. Keisler's paper [22] contains a good introduction to this topic and many related things.

\section{3. א-injective Banach spaces}

In this section we extend some results proved in [4] for separably injective Banach spaces. Recall that a Banach space $E$ is separably injective $\left(\aleph_{1}\right.$-injective according to Definition 1.1) when $E$-valued operators extends to separable super-spaces, and that $E$ is universally separably injective (universally $\aleph_{1}$-injective) when $E$-valued operators extend from separable subspaces. Our first result generalizes Proposition 3.2 in [4].

Proposition 3.1. For a Banach space $E$ and a cardinal $\aleph$, the following assertions are equivalent:

(1) E is ふ-injective.

(2) For every cardinal $\kappa<\aleph$, every operator from a subspace of $\ell_{1}(\kappa)$ into $E$ extends to $\ell_{1}(\kappa)$.

(3) For every Banach space $X$ and each subspace $Y$ such that $\operatorname{dens}(X / Y)<\aleph$, every operator $t: Y \rightarrow E$ extends to $X$.

(4) If $X$ is a Banach space containing $E$ and $\operatorname{dens}(X / E)<\aleph$, then $E$ is complemented in $X$.

Proof. It is clear that $(3) \Rightarrow(1) \Rightarrow(2)$. To show that $(2) \Rightarrow(4)$, observe that if $\operatorname{dens}(X / E)<\aleph$ then there is a quotient map $q: \ell_{1}(\kappa) \rightarrow X / E$ for some $\kappa<\aleph$. 
The operator $q$ can be lifted to an operator $Q: \ell_{1}(\kappa) \rightarrow X$ whose restriction $Q_{0}$ to $\operatorname{ker} q$ actually takes values in $E$. One therefore has a commutative diagram

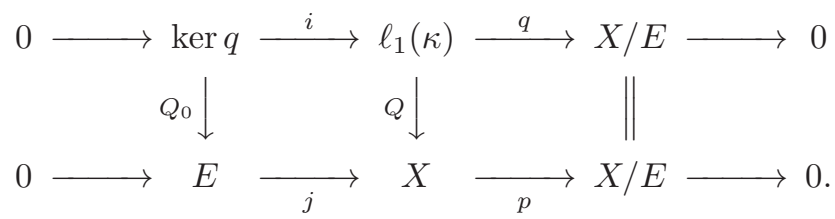

By (2), there is a linear continuous extension $Q_{1}: \ell_{1}(\kappa) \rightarrow E$ of $Q_{0}$. Since $\left(Q-j Q_{1}\right) i=0$, there is an operator $\nu: X / E \rightarrow X$ such that $\nu q=Q-j Q_{1}$. Since $p \nu q=q$, the expression $P=\mathbf{1}_{X}-\nu p$ defines a projection on $X$ onto the subspace $E$.

Now, to show that $(4) \Rightarrow(3)$ just form the push-out diagram

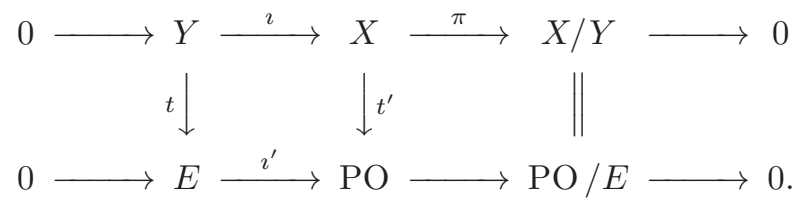

Since $\mathrm{PO} / E=X / Y$, the cardinality assumption is preserved and $E$ must be complemented in $\mathrm{PO}$ by a projection $P$. Thus, $P t^{\prime}$ yields an extension of $t$ as required.

Our next result yields a homological characterization of $\left(2^{\aleph}\right)^{+}$-injectivity:

Proposition 3.2. A Banach space $E$ is $\left(2^{\aleph}\right)^{+}$-injective if and only if it is complemented in every superspace $W$ such that $W / E$ is a quotient of $\ell_{\infty}(\aleph)$.

Proof. Every quotient of $\ell_{\infty}(\aleph)$ has density character at most $2^{\aleph}$; so the necessity is clear by (4) in the preceding proposition.

We prove now the sufficiency as follows: we will show that $E$-valued operators from subspaces of $\ell_{\infty}(\aleph)$ can be extended to the whole $\ell_{\infty}(\aleph)$; which in combination with the fact that $\ell_{1}\left(2^{\aleph}\right)$ is a subspace of $\ell_{\infty}(\aleph)$ and Proposition 3.1 (2) provides the result. Thus, let $t: Z \rightarrow E$ be an operator defined on a subspace $Z$ of $\ell_{\infty}(\aleph)$. One thus gets a push-out diagram

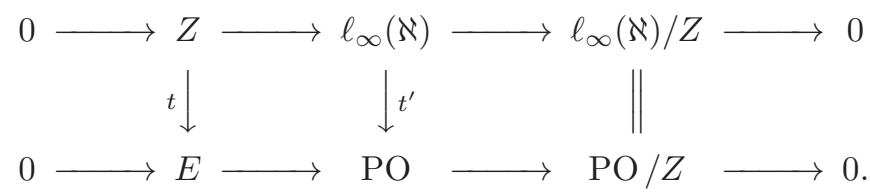

By hypothesis, $E$ is complemented in $\mathrm{PO}$ and thus there is a linear continuous projection $P: \mathrm{PO} \rightarrow E$. The operator $P t^{\prime}: \ell_{\infty}(\aleph) \rightarrow E$ extends $t$. Thus, it has been shown that every operator $t: Z \rightarrow E$ from a subspace of $\ell_{\infty}(\aleph)$ can be extended to the whole $\ell_{\infty}(\aleph)$. To finish the proof it remains to prove the following result; probably it is known, but we were unable to find an explicit reference:

Claim: $\ell_{1}\left(2^{\aleph}\right)$ is a subspace of $\ell_{\infty}(\aleph)$. 
Proof of the claim. The dual ball of $\ell_{1}\left(2^{\aleph}\right)$ in its weak*-topology is homeomorphic to the product $[-1,1]^{2^{\aleph}}$ which is a continuous image of $\{0,1\}^{\aleph^{\aleph}}$. This space has density $\aleph$, as we show now: observe that subsets of $2^{\aleph}$ can be interpreted as elements of $\{0,1\}^{2^{\aleph}}$ via their characteristic functions. Let us show that with this interpretation the clopen sets of $2^{\aleph}$ form a dense set of $\{0,1\}^{2^{\aleph}}$ : take a basic open set $U$; i.e., take points $p_{1}, \ldots, p_{n}$ and $q_{1}, \ldots, q_{m}$ from $2^{\aleph}$ and form the basic open set

$$
U=\left\{x \in\{0,1\}^{2^{\aleph}}: x_{p_{i}}=1 \text { and } x_{q_{i}}=0\right\} .
$$

Find a clopen $C$ of $2^{\aleph}$ such that $p_{1}, \ldots, p_{n}$ are in $C$, but $q_{1}, \ldots, q_{n}$ do not belong to $C$. The characteristic function $1_{C} \in U$. Thus, since $2^{\aleph}$ has $\aleph$ many clopens, the dual ball of $\ell_{1}\left(2^{\aleph}\right)$ in its weak*-topology has density $\aleph$; and thus $\ell_{1}\left(2^{\aleph}\right)$ in its weak ${ }^{*}$-topology has density $\aleph$; and therefore $\ell_{1}\left(2^{\aleph}\right)$ can be embedded into $\ell_{\infty}(\aleph)$, and the claim is proved.

The stability properties of the classes of (universally) $\aleph$-injective spaces are gathered in the following proposition (compare to Proposition 3.7 in [4]).

Proposition 3.3. Let $\aleph$ be an infinite cardinal.

(1) The class of $\aleph$-injective spaces has the 3 -space property.

(2) The quotient of an $\aleph$-injective space by an $\aleph$-injective subspace is $\aleph$-injective.

(3) If $\varkappa \leq \aleph$, the quotient of a universally $\aleph$-injective space by a $\varkappa$-injective subspace is universally $\varkappa$-injective.

Proof. The proof of (1) follows from part (2) in Proposition 3.1: let us consider an exact sequence $0 \longrightarrow F \stackrel{j}{\longrightarrow} E \stackrel{\pi}{\longrightarrow} G \longrightarrow 0$ in which both $F$ and $G$ are $\aleph$-injective. Let $\phi: K \rightarrow E$ be an operator from a subspace $K$ of $\ell_{1}(\kappa)$ with $\kappa<\aleph$, and let $\imath: K \rightarrow \ell_{1}(\kappa)$ denote the natural embedding; then $\pi \phi$ can be extended to an operator $\Phi: \ell_{1}(\kappa) \rightarrow G$, which can in turn be lifted to an operator $\Psi: \ell_{1}(\kappa) \rightarrow E$. The difference $\phi-\Psi \imath$ takes values in $F$ and can thus be extended to an operator $e: \ell_{1}(\kappa) \rightarrow F$. The desired operator is $\Psi+j e$.

To prove (2) let us consider an exact sequence $0 \longrightarrow F \longrightarrow E \stackrel{\pi}{\longrightarrow} G \longrightarrow 0$ in which both $F$ and $E$ are $\aleph$-injective. Let $\phi: Y \rightarrow G$ be an operator from a subspace $Y$ of a space $X$ with dens $X<\aleph$. Consider the pull-back diagram

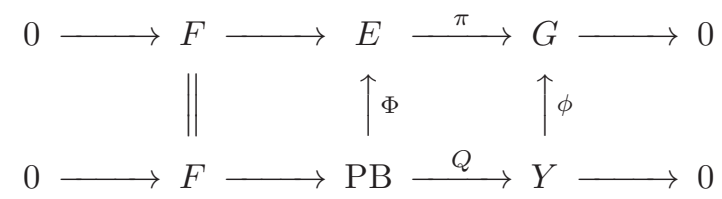

and observe that since $F$ is $\aleph$-injective, the lower exact sequence splits, so $Q$ admits a linear continuous selection $s: Y \rightarrow \mathrm{PB}$. By the $\aleph$-injectivity of $E$, there exists an operator $T: X \rightarrow E$ agreeing with $\Phi s$ on $Y$. Then $\pi T: X \rightarrow G$ is the desired extension of $\phi$ since $\left.\pi T\right|_{Y}=\pi \Phi s=\phi Q s=\phi$.

To prove (3), assume that $E$ is universally $\aleph$-injective and $F$ is $\varkappa$-injective. The previous proof, reproduced verbatim, shows that $G$ is universally $\varkappa$-injective. 
It is perhaps worth to remark that an abstract homological proof that all properties having the form $\operatorname{Ext}(X,-)=0$ are 3 -space properties can be found in [8]. The connection with Proposition 3.3 (1) is that Proposition 3.1 (4) can be read in this language: a Banach space $E$ is $\aleph$-injective if and only if it verifies $\operatorname{Ext}(F, E)=0$ for all Banach spaces $F$ with dens $F<\aleph$.

It would be interesting to know whether the class of universally $\aleph$-injective spaces enjoys the 3 -space property. This is not known even for $\aleph=\aleph_{1}$. It was claimed in Proposition 5.3 of [4] that universal separable injectivity is a 3 -space property; but the "proof" contains a gap we have been unable to fill. Consequently, other claims also remain without proper justification, namely Propositions 5.4 and 5.6 and Theorem 5.5 in [4], and Example 4.5(a) and the second part of Proposition 5.1 in [5]. All other statements in [4] and [5] are independent from Proposition 5.3 in [4]. Note that universally $\aleph$-injectivity is equivalent with the property of $\ell_{\infty}$-super-saturation: every separable subspace of $E$ is contained in a copy of $\ell_{\infty}$ contained in $E$; see Proposition 5.2 in [4]. This characterization could be useful, but apparently there is no higher cardinal analogue for such property. Indeed, the obvious extension fails because there exist injective Banach spaces with arbitrarily large density character, like the spaces $L_{\infty}(\mu)$ for finite $\mu$, that do not contain subspaces isomorphic to $\ell_{\infty}\left(\aleph_{1}\right)$-this is so since a family of mutually disjoint sets of positive measure on a finite measure space must be countable. We can obtain a partial analogue introducing the following concept.

Definition 3.4. Let $\aleph$ be an infinite cardinal. We say that a subspace $Y$ of a Banach space $X$ if $c_{0}(\aleph)$-supplemented if there exists another subspace $Z$ of $X$ isomorphic to $c_{0}(\aleph)$ such that $Y \cap Z=0$ and the sum $Y+Z$ is closed. In this case we will also say that $Z$ is a $c_{0}(\aleph)$-supplement of $Y$.

Lemma 3.5. Each subspace of $\ell_{\infty}(\aleph)$ with density character $\leq \aleph$ is $c_{0}(\aleph)$-supplemented.

Proof. Let $I$ have cardinality $\aleph$ and let $\left\{I_{j}: j \in J\right\}$ be a family of disjoint subsets of $I$ with $\left|I_{j}\right|=\aleph$ for every $j$ and $|J|=\aleph$. Let $Y$ be a subspace of $\ell_{\infty}(I)$ with density character $\leq \aleph$. Since dens $\left(\ell_{\infty}\left(I_{j}\right)\right)>\aleph$, for each $j \in J$ we can find $x_{j} \in \ell_{\infty}\left(I_{j}\right)$ with $\left\|x_{j}\right\|=1$ and $\operatorname{dist}\left(x_{j}, Y\right)>1 / 2$. In this way we obtain a family $\left\{x_{j}: j \in J\right\}$ in $\ell_{\infty}(I)$ isometrically equivalent to the basis of $c_{0}(I)$. Let $\pi: \ell_{\infty}(I) \rightarrow \ell_{\infty}(I) / Y$ denote the quotient map. Since $\inf \left\{\left\|\pi\left(x_{j}\right)\right\|: j \in J\right\} \geq 1 / 2>0$, by Theorem 3.4 in [34] there exists $J_{1} \subset J$ with $\left|J_{1}\right|=|J|$ such that the restriction of $\pi$ to the closed subspace generated by $\left\{x_{j}: j \in J\right\}$ is an isomorphism. That space is a $c_{0}(\aleph)$-supplement of $Y$.

We thus get the partial extension result announced above.

Theorem 3.6. Let $X$ be a universally $\aleph^{+}$-injective Banach space and let $Y$ be a $c_{0}(\aleph)$-supplemented subspace of $X$ with $\operatorname{dens}(Y) \leq \aleph$. Then $Y$ is contained in a subspace of $X$ isomorphic to $\ell_{\infty}(\aleph)$.

Proof. Let $Y_{0}$ be a subspace of $\ell_{\infty}(\aleph)$ isomorphic to $Y$ and let $t: Y_{0} \rightarrow Y$ be a (bijective) isomorphism with $\left\|t^{-1}\right\|=1$. By Lemma 3.5, $Y_{0}$ is $c_{0}(\aleph)$-supplemented 
in $\ell_{\infty}(\aleph)$. We can find projections $P$ on $X$ and $Q$ on $\ell_{\infty}(\aleph)$ such that $Y \subset \operatorname{ker} P$, $Y_{0} \subset \operatorname{ker} Q$, and both ranges $\operatorname{ran} P$ and $\operatorname{ran} Q$ are isomorphic to $\ell_{\infty}(\aleph)$.

Indeed, let $\pi: X \rightarrow X / Y$ be the quotient map. Let $L_{0}$ and $L$ denote $c_{0}(\aleph)$ supplements for $Y_{0}$ and $Y$. The isomorphism $t: Y_{0} \rightarrow Y$ admits an extension $\hat{t}: Y_{0} \oplus L_{0} \rightarrow Y \oplus L$, and the universal $\aleph^{+}$-injectivity of $X$ allows us to find an operator $I: \ell_{\infty}(\aleph) \rightarrow X$ extending $\hat{t}$, hence $\pi I$ is an isomorphism on a copy $L_{0}$ of $c_{0}(\aleph)$. A classical result of Rosenthal (Theorem 1.3 in [34]) yields that $\pi I$ is also an isomorphism on a copy $M_{0}$ of $\ell_{\infty}(\aleph)$ inside $\ell_{\infty}(\aleph)$. Therefore $M=I\left(M_{0}\right)$ is a subspace of $X$ isomorphic to $\ell_{\infty}(\aleph)$ where the restriction of $\pi$ is an isomorphism. Now $X / Y=\pi(M) \oplus N$, with $N$ a closed subspace. Hence $X=M \oplus \pi^{-1}(N)$, and it is enough to take as $P$ the projection with range $M$ and kernel $\pi^{-1}(N)$. Similarly, the quotient map $\pi_{0}: \ell_{\infty}(\aleph) \rightarrow \ell_{\infty}(\aleph) / Y_{0}$ is an isomorphism on $L_{0}$, and Rosenthal's result implies that $\pi_{0}$ is an isomorphism on a subspace $M_{0}$ of $\ell_{\infty}(\aleph)$ isomorphic to $\ell_{\infty}(\aleph)$. Thus $\ell_{\infty}(\aleph) / Y_{0}=\pi_{0}\left(M_{0}\right) \oplus N_{0}$, with $N_{0}$ a closed subspace. Hence $\ell_{\infty}(\aleph)=M_{0} \oplus \pi_{0}^{-1}\left(N_{0}\right)$, and it is enough to take as $Q$ the projection with range $M_{0}$ and kernel $\pi_{0}^{-1}\left(N_{0}\right)$.

Since $\operatorname{ker} P$ and $\operatorname{ker} Q$ are universally $\aleph$-injective spaces, there are operators $U: X \rightarrow \operatorname{ker} Q$ and $V: \ell_{\infty}(\aleph) \rightarrow \operatorname{ker} P$ such that $\left.V\right|_{Y_{0}}=t$ and $\left.U\right|_{Y}=t^{-1}$. Note that $\|U\| \geq 1$. Let $W: \ell_{\infty}(\aleph) \rightarrow \operatorname{ran} P$ be an operator satisfying $\|W x\| \geq\|x\|$ for all $x$. We will show that the operator

$$
T=V+W\left(\mathbf{1}_{\ell_{\infty}(\aleph)}-U V\right): \ell_{\infty}(\aleph) \longrightarrow X
$$

is an isomorphism (into). Since $\operatorname{ran} V \subset \operatorname{ker} P$ and $\operatorname{ran} W \subset \operatorname{ran} P$, there exists $C>0$ such that

$$
\|T x\| \geq C \max \left\{\|V x\|,\left\|W\left(\mathbf{1}_{\ell_{\infty}(\aleph)}-U V\right) x\right\|\right\}
$$

for every $x \in \ell_{\infty}(\aleph)$. Now, if $\|V x\|<(2\|U\|)^{-1}\|x\|$, then $\|U V x\|<(1 / 2)\|x\|$; hence

$$
\left\|W\left(\mathbf{1}_{\ell_{\infty}(\aleph)}-U V\right) x\right\| \geq\left\|\left(\mathbf{1}_{\ell_{\infty}(\aleph)}-U V\right) x\right\|>\|x\| / 2 .
$$

Thus $\|T x\| \geq C(2\|U\|)^{-1}\|x\|$ for every $x \in X$; hence $Y$ is contained in the range of $T$, which is isomorphic to $\ell_{\infty}(\aleph)$.

By the Lindenstrauss-Rosenthal theorem [26] any isomorphism between two separable subspaces of $\ell_{\infty}$ can be extended to an automorphism of $\ell_{\infty}$. As a consequence of Theorem 3.6 we can prove that universally $\aleph^{+}$-injective spaces enjoy a similar property.

Theorem 3.7. Let $X$ be a universally $\aleph^{+}$-injective Banach space, and let $Y_{1}$ and $Y_{2}$ be isomorphic $c_{0}(\aleph)$-supplemented subspaces of $X$ with $\operatorname{dens}\left(Y_{i}\right) \leq \aleph$. Then every isomorphism from $Y_{1}$ onto $Y_{2}$ extends to an automorphism of $X$.

Proof. Note that we can modify the proof of Theorem 3.6 in such a way that the subspace $Z$ isomorphic to $\ell_{\infty}(\aleph)$ that contains $Y$ has a complement isomorphic to $X$. Indeed, if we write $\operatorname{ran}(P)$ as the direct sum of two copies of $\ell_{\infty}(\aleph)$ and 
take $W$ so that its image is contained in one of the summands, then the complement $Z^{\prime}$ of $Z$ in $X$ contains a subspace isomorphic to $\ell_{\infty}(\aleph)$; hence

$$
Z^{\prime} \sim Z^{\prime \prime} \oplus \ell_{\infty}(\aleph) \sim Z^{\prime \prime} \oplus \ell_{\infty}(\aleph) \oplus \ell_{\infty}(\aleph) \sim Z^{\prime} \oplus \ell_{\infty}(\aleph) \sim Z^{\prime} \oplus Z \sim X .
$$

So, for each $i=1,2$, we can assume that $Y_{i}$ is contained in a subspace $Z_{i}$ isomorphic to $\ell_{\infty}(\aleph)$ such that the complement of $Z_{i}$ in $X$ is isomorphic to $X$. Therefore, given an isomorphism $T: Y_{1} \rightarrow Y_{2}$, since the quotients $Z_{1} / Y_{1}$ and $Z_{2} / Y_{2}$ are not reflexive, we first extend $T$ to an isomorphism $\tau$ from $Z_{1}$ onto $Z_{2}$, which clearly can be extended to an automorphism of $X$.

The extension from $T: Y_{1} \rightarrow Y_{2}$ to $\tau: Z_{1} \rightarrow Z_{2}$ can be obtained as in the proof of part (i) of Theorem 2.f.12 in [27]. First, as in the proof of Theorem 3.6, we get projections $P_{i}$ on $Z_{i}(i=1,2)$ with $Y_{i} \subset \operatorname{ker} P_{i}$ and both $\operatorname{ker} P_{i}$ and $\operatorname{ran} P_{i}$ isomorphic to $\ell_{\infty}(\aleph)$.

Since the space $\operatorname{ran}\left(\mathbf{1}_{Z_{2}}-P_{2}\right)$ is injective and contains $Y_{2}$, there exists an extension $S_{1}: Z_{1} \rightarrow \operatorname{ran}\left(\mathbf{1}_{Z_{2}}-P_{2}\right)$ of $T$, and similarly there is an extension $S_{2}: Z_{2} \rightarrow \operatorname{ran}\left(\mathbf{1}_{Z_{1}}-P_{1}\right)$ of $T^{-1}$. Let $R$ be an isomorphism from $Z_{1}$ onto $\operatorname{ran}\left(P_{2}\right)$ with $\left\|R^{-1}\right\|>1$, and define $\hat{T}: Z_{1} \rightarrow Z_{2}$ by $\hat{T}=S_{1}+R\left(\mathbf{1}_{Z_{1}}-S_{2} S_{1}\right)$. Since $\left.\left(\mathbf{1}_{Z_{1}}-S_{2} S_{1}\right)\right|_{Y_{1}}=0$ it follows that $\hat{T}$ is an extension of $T$, and as in the proof of part (i) of Theorem 2.f.12 in [27], we can check that $\hat{T}$ is an into isomorphism.

Note that the subspace $\hat{T}\left(\operatorname{ran}\left(\mathbf{1}_{Z_{1}}-P_{1}\right)\right)$ is complemented in $Z_{2}$ with complement $W$ isomorphic to $Z_{2}$. Let $R_{0}$ be an isomorphism from $\operatorname{ran} P_{1}$ onto $W$. The operator $\tau=R_{0} P_{1}+\hat{T}\left(\mathbf{1}_{Z_{1}}-P_{1}\right)$ is an isomorphism from $Z_{1}$ onto $Z_{2}$ extending $T$.

Further differences between $\aleph$-injectivity and separable injectivity is that Sobczyk's theorem has no simple counterpart for higher cardinals: indeed, $c_{0}(\aleph)$ is never $\aleph_{2}$-injective just because its complemented subspace $c_{0}$ is not: recall from [4] and [9] the existence of the Johnson-Lindenstrauss nontrivial exact sequences

$$
0 \longrightarrow c_{0} \longrightarrow J L \longrightarrow c_{0}\left(\aleph_{1}\right) \longrightarrow 0
$$

Perhaps the role of $c_{0}$ could be played by Hasanov's "filter version" of $c_{0}$ (see [17]). Recall that a filter $\mathscr{F}$ on a set $S$ is called $\aleph$-complete if whenever $A_{i} \in \mathscr{F}$ for all $i \in I$ with $|I|<\aleph$ then $\bigcap_{i \in I} A_{i}$ is again in $\mathscr{F}$. The space $c_{0}^{\mathscr{F}}(S)$ is the closed linear span in $\ell_{\infty}(S)$ of the set $\left\{x \in \ell_{\infty}(S): \lim _{\mathscr{F}} x=0\right\}$. Hasanov shows in [17] that if $\mathscr{F}$ is $\aleph$-complete, then $c_{0}^{\mathscr{F}}(S)$ is at most 2-complemented in any superspace $E$ such that $\operatorname{dens}\left(E / c_{0}^{\mathscr{F}}(S)\right) \leq \aleph$. Thus, it is $\left(2, \aleph^{+}\right)$-injective.

\section{4. $(1, \aleph)$-injective Banach spaces}

The $(1, \aleph)$-injective spaces can be characterized as follows (this result can be essentially found as the remark after Corollary 2, p. 56, in [24]; the "if part" is due to Aronszajn and Panitchpakdi, see Theorem 3 in [2]).

Lemma 4.1. A Banach space $E$ is $(1, \aleph)$-injective if and only if every family of less than $\aleph$ mutually intersecting balls of $E$ has nonempty intersection.

Proof. Sufficiency. Take an operator $t: Y \rightarrow E$, where $Y$ is a closed subspace of $X$, where dens $X<\aleph$. We may and do assume $\|t\|=1$. Let $z \in X \backslash Y$ and let $Y_{0}$ 
be a dense subset of $Y$ forming a linear space over the rational numbers with $\left|Y_{0}\right|<\aleph$ and, for each $y \in Y_{0}$, consider the ball $B(t y,\|y-z\|)$ in $E$. Any two of these balls intersect, since for $y_{1}, y_{2} \in Y_{0}$ we have

$$
\left\|t y_{2}-t y_{1}\right\| \leq\|t\|\left\|y_{2}-y_{1}\right\| \leq\left\|y_{2}-z\right\|+\left\|y_{1}-z\right\| \text {. }
$$

The hypothesis is that there is

$$
f \in \bigcap_{y \in Y_{0}} B(t y,\|y-z\|)=\bigcap_{y \in Y} B(t y,\|y-z\|) .
$$

It is clear that the map $T: Y+\langle z\rangle \rightarrow E$ defined by $T(y+c z)=t y+c f$ is an extension of $t$ with $\|T\|=1$. The rest is clear: use Zorn lemma.

NECESSITY. We begin with the observation that if two closed balls of a Banach space have a common point, then the distance between the centers is at most the sum of the radii. In $\ell_{\infty}(\aleph)$ that necessary condition is sufficient. On the other hand, in $\ell_{\infty}(\aleph)$, every family of mutually intersecting balls has nonempty intersection - this is trivial. So, let $E$ be $(1, \aleph)$-injective and suppose $B\left(e_{i}, r_{i}\right)$ is a family of less than $\aleph$ mutually intersecting balls in $E$. Let $Y$ be the closed subspace of $E$ spanned by the centers, so that dens $Y<\aleph$. Let $\jmath: Y \rightarrow \ell_{\infty}(\aleph)$ be any isometric embedding. Notice that even if $B_{Y}\left(e_{i}, r_{i}\right)=B\left(e_{i}, r_{i}\right) \cap Y$ need not be mutually intersecting in $Y$, any two balls of the family $B\left(\jmath\left(e_{i}\right), r_{i}\right)$ meet in $\ell_{\infty}(\aleph)$ because the distance between the centers does not exceed the sum of the radii. Therefore the intersection

$$
\bigcap_{i} B\left(\jmath\left(e_{i}\right), r_{i}\right)
$$

contains some point, say $x \in \ell_{\infty}(\aleph)$. Let $X$ be the subspace spanned by $x$ and $\jmath(Y)$ in $\ell_{\infty}(\aleph)$. The hypothesis on $E$ allows one to extend the inclusion $\imath: Y \rightarrow E$ through $\jmath: Y \rightarrow X$ to an operator $I: X \rightarrow E$ without increasing the norm; i.e., $I \jmath=\imath$. Since $\left\|I(x)-e_{i}\right\|=\left\|I\left(x-\jmath e_{i}\right)\right\| \leq\left\|x-\jmath e_{i}\right\|$ one gets $I(x) \in \bigcap_{i} B\left(e_{i}, r_{i}\right)$.

Proposition 6.2 of [4] asserts that, under $\mathrm{CH}$, 1-separably injective spaces are universally 1-separably injective. This admits a higher cardinal counterpart, which stems from remark 6, p. 223, in [25].

Proposition 4.2. Under $\mathrm{GCH}$, every $(1, \aleph)$-injective Banach space is universally $(1, \aleph)$-injective.

Proof. Let $E$ be an $(1, \aleph)$-injective Banach space and let $Y$ be a density character $\aleph$ subspace of a space $X$ and let $t: Y \rightarrow E$ be an operator. Let $j: Y \rightarrow \ell_{\infty}(\aleph)$ be an isometric embedding; and observe that, under GCH, the space $\ell_{\infty}(\aleph)$ has density character $\aleph^{+}$. Since a set of cardinal $\aleph^{+}$can be written as the union of an increasing chain of sets of cardinal $\aleph$, write $\ell_{\infty}(\aleph)$ as the union of an increasing chain of subspaces with density character $\aleph$. There is no loss of generality in assuming that the first set of the chain is $Y$. Use transfinite induction and the $(1, \aleph)$-injectivity of $E$ to extend $t$ to an operator $T: \ell_{\infty}(\aleph) \rightarrow E$ with the same norm (see Lemma 6.1 in [4] for details). Extend $j$ to an operator $J: X \rightarrow \ell_{\infty}(\aleph)$ with the same norm. The composition $T J$ is the desired equal norm extension of $t$. 


\section{Spaces of continuous functions}

The following omnibus result summarizes what is known about the interplay between the $(1, \aleph)$-injectivity of $C(K)$, the topological properties of $K$ and the lattice structure of $C(K)$. We will need a simple lemma which can be found in [4] (Lemma 6.4).

Lemma 5.1. Let $K, L$ and $M$ be compact spaces and let $f: K \rightarrow M$ be a continuous map, with $\jmath=f^{\circ}: C(M) \rightarrow C(K)$ its induced operator, and let $\imath: C(M) \rightarrow$ $C(L)$ be a positive norm one operator. Suppose that $S: C(L) \rightarrow C(K)$ is an operator with $\|S\|=1$ and $S \imath=$ J. Then $S$ is a positive operator.

Recall that a cozero set in a topological space $K$ is an open set of the form $\{x \in K: f(x) \neq 0\}$ for some $f \in C(K)$. One has:

Theorem 5.2. For a compact space $K$ and a cardinal number $\aleph \geq \aleph_{1}$, the following statements are equivalent:

(a) $C(K)$ is $(1, \aleph)$-injective.

(b) Given subsets $L$ and $U$ of $C(K)$ with $|L|,|U|<\aleph$ such that $f \leq g$ for every $f \in L$ and $g \in U$, there exists $h \in C(K)$ separating them, that is, such that $f \leq h \leq g$ for all $f \in L$ and $g \in U$.

(c) Every family of mutually intersecting balls in $C(K)$ of cardinal less than has nonempty intersection.

(d) Every couple of disjoint open sets $G$ and $H$ of $K$ which are the union of less than $\aleph$ many closed sets have disjoint closures.

(e) Every couple of disjoint open sets $G$ and $H$ of $K$ which are the union of less than $\aleph$ many cozero sets have disjoint closures.

Proof. We first prove the implications (a) $\Rightarrow(\mathrm{b}) \Rightarrow(\mathrm{c}) \Rightarrow(\mathrm{a})$, in that order. Let $L$ and $U$ be as in (b). We consider $C(K)$ as a subalgebra of $\ell_{\infty}(K)$. Let $\eta \in \ell_{\infty}(K)$ such that $f \leq \eta \leq g$ for all $f \in L$ and $g \in U$. Let $A$ be the least unital closed subalgebra of $\ell_{\infty}(K)$ containing $L, U$ and $\eta$, and let $B=A \cap C(K)$. Clearly, dens $A<\aleph$. By (a), the inclusion of $B$ into $C(K)$ extends to a norm-one operator $I: A \rightarrow C(K)$. Let $L$ be the maximal ideal space of $A$ and $M$ that of $B$. By general representation theorems we have $A=C(L), B=C(M)$ (see for instance Theorem 4.2.5 in [1]) and a commutative diagram

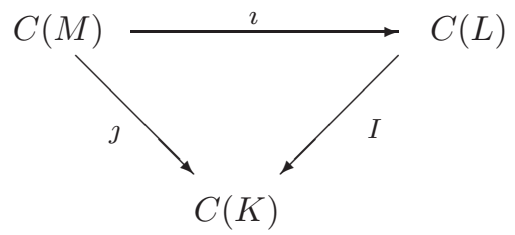

By Lemma 5.1, $I$ is a positive operator, hence $I \eta$ separates $L$ from $U$. 
We check now $(\mathrm{b}) \Rightarrow(\mathrm{c})$. Let $\left(B_{i}\right)_{i \in I}$ be a family of mutually intersecting balls, where $|I|<\aleph$. Writing $B_{i}=B\left(f_{i}, r_{i}\right)$, we have $\left\|f_{i}-f_{j}\right\| \leq r_{i}+r_{j}$ for all $i, j \in I$, that is,

$$
f_{i}-r_{i} \leq f_{j}+r_{j} \quad(i, j \in I) .
$$

By (b) there is $h \in C(K)$ such that

$$
f_{i}-r_{i} \leq h \leq f_{j}+r_{j} \quad(i, j \in I) .
$$

In particular $f_{i}-r_{i} \leq h \leq f_{i}+r_{i}$, that is, $h \in \bigcap_{i} B_{i}$. The implication $(c) \Rightarrow(a)$ is contained in Lemma 4.1 .

We pass to the string (b) $\Rightarrow(\mathrm{d}) \Rightarrow(\mathrm{e}) \Rightarrow(\mathrm{b})$. Assume that (b) holds and let $G$ and $H$ be as in (d), so that $G=\bigcup_{\alpha \in I} C_{\alpha}$ and $H=\bigcup_{\alpha \in I} D_{\alpha}$, where $C_{\alpha}$ and $D_{\alpha}$ are closed subsets of $K$ and $|I|<\aleph$. For every $\alpha \in I$, let $f_{\alpha} \in C(K), 0 \leq f_{\alpha} \leq 1$, such that $\left.f_{\alpha}\right|_{K \backslash G}=0$ and $\left.f_{\alpha}\right|_{C_{\alpha}}=1$, and let $g_{\alpha} \in C(K), 0 \leq g_{\alpha} \leq 1$, such that $\left.g_{\alpha}\right|_{K \backslash H}=1$ and $\left.g_{\alpha}\right|_{D_{\alpha}}=0$. The sets $L=\left\{f_{\alpha}: \alpha \in I\right\}$ and $U=\left\{g_{\alpha}: \alpha \in I\right\}$ satisfy the assumptions of condition (b). The function $h \in C(K)$ that separates $L$ and $U$ has the property that $\left.h\right|_{G}=1$ and $\left.h\right|_{H}=0$, hence $\bar{G} \cap \bar{H}=\varnothing$. That (d) implies (e) is a consequence of the fact that each cozero set is the union of countably many closed sets, namely for $f \in C(K)$,

$$
\{x \in K: f(x) \neq 0\}=\bigcup_{n \in \mathbb{N}}\{x \in K:|f(x)| \geq 1 / n\} .
$$

Assume now that (e) holds. As a first step towards (b), we prove it modulo a given positive $\varepsilon$.

Claim. Given $U$ and $L$ like in (b) and given $\varepsilon>0$, there exists $h \in C(K)$ such that $f-\varepsilon \leq h \leq g+\varepsilon$ for every $f \in L$ and $g \in U$.

Proof of the claim. By homogeneity, it is enough to consider the case $\varepsilon=1$. Let $N \in \mathbb{N}$ be such that $-N<f_{0} \leq g_{0}<N$ for some $f_{0} \in L$ and $g_{0} \in U$. Let $I=\{n \in \mathbb{N}:-N<n<N\}$. For every $n \in I$, let

$$
\begin{aligned}
& G_{n}=\{x \in K: f(x)>n \text { for some } f \in L\}=\bigcup_{f \in L} f^{-1}(n,+\infty), \\
& H_{n}=\{x \in K: g(x)<n \text { for some } g \in U\}=\bigcup_{g \in U} g^{-1}(-\infty, n) .
\end{aligned}
$$

For each $n, G_{n}$ and $H_{n}$ are disjoint open sets which are the union of less than cozero sets, because $|L|,|U|<\aleph$ and $f^{-1}(n,+\infty)$ is itself a cozero set (it is the complement of the zero set of $\max \{f-n, 0\})$. Hence $\overline{G_{n}} \cap \overline{H_{n}}=\varnothing$, therefore there exists $h_{n} \in C(K),-1 \leq h_{n} \leq 1$ such that $\left.h_{n}\right|_{G_{n}}=1$ and $\left.h_{n}\right|_{H_{n}}=-1$. We shall check that $h=\frac{1}{2} \sum_{n \in I} h_{n} \in C(K)$ is the desired function. For $f \in L$ and $x \in K$,

$$
\begin{aligned}
h(x) & =\frac{1}{2} \sum_{n \in I} h_{n}(x)=\frac{1}{2}\left(\sum_{n \in I, n<f(x)}(1)+\sum_{n \in I, n \geq f(x)} h_{n}(x)\right) \\
& \geq \frac{|\{n \in I, n<f(x)\}|-|\{n \in I, n \geq f(x)\}|}{2} \geq f(x)-1 .
\end{aligned}
$$

Similarly, one gets that $h(x) \leq g(x)+1$ for all $g \in U$ and $x \in K$. 
Now, if $U$ and $L$ are sets like in (b) we construct inductively a sequence of new sets $U_{n}, L_{n} \subset C(K)$ and functions $h_{n} \in C(K)$ as follows: $L_{0}=L, U_{0}=U$; $h_{n} \in C(K)$ is such that $f-2^{-n} \leq h_{n} \leq g+2^{-n}$ for all $f \in L_{n}, g \in U_{n}$; $L_{n+1}=L_{n} \cup\left\{h_{n}-2^{-n}\right\}, U_{n+1}=U_{n} \cup\left\{h_{n}+2^{-n}\right\}$. This can be performed because of the preceding claim. Notice that the sequence $\left(h_{n}\right)_{n \in \mathbb{N}}$ is uniformly convergent because for $m<n, h_{m}-2^{-m} \in L_{n}, h_{m}+2^{-m} \in U_{n}$, hence

$$
h_{m}-2^{-m}-2^{-n} \leq h_{n} \leq h_{m}+2^{-m}+2^{-n} \Rightarrow\left\|h_{n}-h_{m}\right\| \leq 2^{-m+1} .
$$

We can consider thus $h=\lim _{n} h_{n}$. This function belongs to $C(K)$ and satisfies $f \leq h \leq g$ for $f \in L$ and $g \in U$.

Remark 5.3. The preceding theorem summarizes or generalizes many earlier results. The equivalence between (a), (d) and (e) can be traced back to Theorem 2 in [2], although Aronszajn and Panitchpakdi manage a condition intermediate between (d) and (e); see also Henriksen's note [21]. Neville's Theorem 2 in [31] is clearly related to the equivalence between (a) and (b). The equivalence between (b) and (e) when $\aleph=\aleph_{1}$ if due to Seever (see Theorem 2.5 in [35]).

As a consequence of Theorem 5.2 we get (cf. Section 4.3 in [4]):

Proposition 5.4. The space $C\left(\mathbb{N}^{*}\right)=\ell_{\infty} / c_{0}$ is $\left(1, \aleph_{1}\right)$-injective.

Recall that $\mathbb{N}^{*}=\beta \mathbb{N} \backslash \mathbb{N}$. We show now that no cardinal improvement is possible.

Proposition 5.5. $C\left(\mathbb{N}^{*}\right)$ is not $\left(1, \aleph_{2}\right)$-injective.

Proof. A classical construction in set theory known as the Hausdorff gap [18] yields the existence of two $\omega_{1}$-sequences of clopen sets in $\mathbb{N}^{*}$, say $\left(a_{i}\right)$ and $\left(b_{i}\right)$ where $i \in \omega_{1}$, such that $\left(a_{i}\right)$ is increasing, $\left(b_{i}\right)$ is decreasing, $a_{i} \subset b_{j}$ for all $i, j$ and with the additional property that for no clopen set $c$ one may have $a_{i} \subseteq c \subseteq b_{j}$ for all $i, j \in \omega_{1}$. Considering the characteristic functions of those clopen sets, condition (b) of Theorem 5.2 is violated for $\aleph=\aleph_{2}$ (take into account that zerodimensional compacta are in fact strongly zero-dimensional, that is, disjoint zero sets can be put into disjoint clopen sets).

If we deal with $\aleph$-injectivity instead of $(1, \aleph)$-injectivity, the matter becomes more complicated: since $C\left(\mathbb{N}^{*}\right)$ contains an uncomplemented copy of itself $[10]$ it is not $\mathfrak{c}^{+}$-injective. We do not know whether it is consistent that $C\left(\mathbb{N}^{*}\right)$ is $\aleph_{2}$-injective.

\section{Ultraproducts}

Perfect examples of compact spaces as those of Theorem 5.2 - the objects that we will study in the next section - can be obtained via ultraproducts. Let us briefly recall the definition and some basic properties of ultraproducts of Banach spaces. For a detailed study of this construction at the elementary level needed here we refer the reader to Heinrich's survey paper [19] or Sims' notes [36]. 
Let $\left(X_{i}\right)_{i \in I}$ be a family of Banach spaces indexed by $I$ and let $\mathscr{U}$ be an ultrafilter on $I$. The space $\ell_{\infty}\left(X_{i}\right)$ endowed with the supremum norm is a Banach space, and $c_{0}^{\mathscr{U}}\left(X_{i}\right)=\left\{\left(x_{i}\right) \in \ell_{\infty}\left(X_{i}\right): \lim _{\mathscr{U}(i)}\left\|x_{i}\right\|=0\right\}$ is a closed subspace of $\ell_{\infty}\left(X_{i}\right)$. The ultraproduct of the spaces $\left(X_{i}\right)_{i \in I}$ following $\mathscr{U}$ is defined as the quotient

$$
\left[X_{i}\right]_{\mathscr{U}}=\ell_{\infty}\left(X_{i}\right) / c_{0}^{\mathscr{U}}\left(X_{i}\right),
$$

with the quotient norm. We denote by $\left[\left(x_{i}\right)\right]$ the element of $\left[X_{i}\right]_{\mathscr{U}}$ which has the family $\left(x_{i}\right)$ as a representative. It is not difficult to show that $\left\|\left[\left(x_{i}\right)\right]\right\|=$ $\lim _{\mathscr{U}(i)}\left\|x_{i}\right\|$. In the case $X_{i}=X$ for all $i$, we denote the ultraproduct by $X_{\mathscr{U}}$, and call it the ultrapower of $X$ following $\mathscr{U}$.

There is an obvious connection between $\left[X_{i}\right]_{\mathscr{U}}$ and the set-theoretic ultraproduct $\left\langle X_{i}\right\rangle_{U}$ defined in Subsection 2.5: indeed, the former space can be obtained from the latter, first taking the elements for which the seminorm

$$
\left\langle\left(x_{i}\right)\right\rangle_{\mathscr{U}} \longmapsto \lim _{\mathscr{U}(i)}\left\|x_{i}\right\|
$$

is finite (we may consider the original norms on the $X_{i}$ as taking values on the extended ray $[0, \infty])$, and then taking quotient by the kernel of the seminorm.

If $\left(X_{i}\right)_{i \in I}$ is a family of Banach algebras, then $\ell_{\infty}\left(X_{i}\right)$ is also a Banach algebra, with the coordinatewise product. Thus, if $\mathscr{U}$ is an ultrafilter on $I, c_{0}^{\mathscr{U}}\left(X_{i}\right)$ is an ideal in $\ell_{\infty}\left(X_{i}\right)$ and $\left[X_{i}\right]_{\mathscr{U}}$ becomes a Banach algebra with product

$$
\left[\left(x_{i}\right)\right] \cdot\left[\left(y_{i}\right)\right]=\left[\left(x_{i} \cdot y_{i}\right)\right] .
$$

Thus, if $\left(K_{i}\right)_{i \in I}$ is a family of compact spaces, the algebra $\left[C\left(K_{i}\right)\right]_{\mathscr{U}}$ is isometrically isomorphic to $C(K)$, for some compact space $K$; see Theorem 4.2.5 in [1]. This compact is called the (topological) ultracoproduct of $\left(K_{i}\right)_{i \in I}$, and it is denoted by $\left(K_{i}\right)^{\mathscr{U}}$; actually $\left(K_{i}\right)^{\mathscr{U}}$ is the maximal ideal space of $\left[C\left(K_{i}\right)\right]_{\mathscr{U}}$ equipped with the relative weak* topology. We refer the reader to Section 5 in [7] for a purely topological description of the ultracoproduct construction, although we will not use it.

An ultrafilter $\mathscr{U}$ on a set $I$ is countably incomplete if here is a decreasing sequence $\left(I_{n}\right)$ of subsets of $I$ such that $I_{n} \in \mathscr{U}$ for all $n$, and $\bigcap_{n=1}^{\infty} I_{n}=\varnothing$.

Notice that $\mathscr{U}$ is countably incomplete if and only if there is a function $n: I \rightarrow \mathbb{N}$ such that $n(i) \rightarrow \infty$ along $\mathscr{U}$ (equivalently, there is a family $\varepsilon(i)$ of strictly positive numbers converging to zero along $\mathscr{U})$. It is obvious that any countably incomplete ultrafilter is non-principal and also that every non-principal (or free) ultrafilter on $\mathbb{N}$ is countably incomplete.

In order to present the main result of the Section we need Keisler's notion of an $\aleph$-good ultrafilter [11], [7].

Definition 6.1. Let $\operatorname{fin}(S)$ denote the set of finite subsets of a given set $S$. If $\mathscr{U}$ is an ultrafilter on $I$, we say that $f: \operatorname{fin}(S) \rightarrow \mathscr{U}$ is monotone (respectively, multiplicative) if $f(A) \supset f(B)$ whenever $A \subset B$ (respectively, if $f(A \cup B)=f(A) \cap f(B)$ ). The ultrafilter $\mathscr{U}$ is said to be $\aleph$-good if, for every $S$ with $|S|<\aleph$, and every monotone $f: \operatorname{fin}(S) \rightarrow \mathscr{U}$, there is a multiplicative $g: \operatorname{fin}(S) \rightarrow \mathscr{U}$ such that $g(A) \subset f(A)$ for all $A$. 
Every set of cardinality $\aleph$ supports $\aleph^{+}$-good ultrafilters (see Theorem 10.4 in [12] or Theorem 6.1.4 in [11]). Every countably incomplete ultrafilter is $\aleph_{1}$-good. Since an $\aleph^{++}$-good ultrafilter on set of cardinality $\aleph$ is necessarily fixed (by saturation and Proposition 4.2.2 in [11]), $\aleph^{+}$-good ultrafilters will be simply called "good ultrafilters".

Theorem 6.2. Let $\mathscr{U}$ be a countably incomplete, $\aleph$-good ultrafilter on $I$ and let $X_{i}$ be a family of Banach spaces indexed by $I$. If $\left[X_{i}\right]_{\mathscr{U}}$ is a Lindenstrauss space, then it is $(1, \aleph)$-injective.

Proof. The key point is the saturation property of the set-theoretic ultraproducts via good ultrafilters. Let $\left(S_{i}\right)_{i \in I}$ be a family of sets and let $\mathscr{U}$ be an ultrafilter on $I$. A subset $A$ of $\left\langle S_{i}\right\rangle_{\mathscr{U}}$ is called internal if there are sets $A_{i} \subset S_{i}$ such that $A=\left\langle A_{i}\right\rangle_{\mathscr{U}}$. It can be proved (see Theorem 4.2.5 in [11] or Theorem 13.9 in [12]) that if $\mathscr{U}$ is countably incomplete and $\aleph$-good, then every family of less than $\aleph$ internal subsets of $\left\langle S_{i}\right\rangle_{\mathscr{U}}$ having the finite intersection property has nonempty intersection.

Let $\left(B^{\alpha}\right)_{\alpha \in \Gamma}$ be a family of mutually intersecting balls in $\left[X_{i}\right]_{\mathscr{U}}$, with $|\Gamma|<\aleph$. Let us write $B^{\alpha}=B\left(x^{\alpha}, r_{\alpha}\right)$ and let $\left(x_{i}^{\alpha}\right)$ be fixed representations of $x^{\alpha}$. Clearly, $\left\langle B\left(x_{i}^{\alpha}, r_{\alpha}+1 / m\right)\right\rangle_{\mathscr{U}}$ is a lifting of $B\left(x^{\alpha}, r_{\alpha}+1 / m\right)$ in the set-theoretic ultraproduct $\left\langle X_{i}\right\rangle_{\mathscr{U}}$. As $\left[X_{i}\right]_{\mathscr{U}}$ is a Lindenstrauss space, the original family $\left(B^{\alpha}\right)$ has the finite intersection property (see the equivalence between (4.12) and (4.13) in Theorem 4.1 of [39]). This implies the same for the family of internal sets

$$
\left(\left\langle B\left(x_{i}^{\alpha}, r_{\alpha}+1 / m\right)\right\rangle_{\mathscr{U}}\right)_{(\alpha, m) \in \Gamma \times \mathbb{N}^{*}}
$$

Indeed, if $F$ is a finite subset of $\Gamma \times \mathbb{N}$, we may assume it is of the form $E \times$ $\{1, \ldots, k\}$ for some finite $E \subset \Gamma$. Then there exists $z \in \bigcap_{\alpha \in E} B^{\alpha}$. Thus, if $\left(z_{i}\right)$ is a representative of $z$, the sets $\left\{i \in I:\left\|x_{i}^{\alpha}-z_{i}\right\| \leq 1 / k\right\}$ belong to $\mathscr{U}$ for every $\alpha \in E$ and $\left\langle\left(z_{i}\right)\right\rangle_{\mathscr{U}} \in \bigcap_{(\alpha, m) \in F}\left\langle B\left(x_{i}^{\alpha}, r_{\alpha}+1 / m\right)\right\rangle_{\mathscr{U}}$.

Since $|\Gamma \times \mathbb{N}|<\aleph$ and $\mathscr{U}$ is $\aleph$-good, there is $x \in\left\langle X_{i}\right\rangle_{\mathscr{U}}$ in the nonempty intersection

$$
\bigcap_{(\alpha, m) \in \Gamma \times \mathbb{N}}\left\langle B\left(x_{i}^{\alpha}, r_{\alpha}+1 / m\right)\right\rangle_{\mathscr{U}} \cdot
$$

It is clear that if $\left(x_{i}\right)$ is any representation of $x$, then

$$
\left[\left(x_{i}\right)\right] \in \bigcap_{\alpha, m} B\left(x^{\alpha}, r_{\alpha}+1 / m\right)=\bigcap_{\alpha \in \Gamma} B^{\alpha},
$$

which completes the proof.

A combination of [4] and [3], see also [5], shows that there exist universally 1-separably injective spaces not isomorphic to any $C(K)$ space. A higher cardinal generalization is as follows.

Example 6.3. For every cardinal $\aleph$ there exists a space of density $2^{\aleph}$ such that

(a) It is $\left(1, \aleph^{+}\right)$-injective but it is not isomorphic to a complemented subspace of any $\mathcal{M}$-space. 
(b) After suitable renorming, it is still $\left(1, \aleph^{+}\right)$-injective and its unit ball has extreme points.

Proof. (a) Let $\mathcal{G}$ be the Gurariu space. If $\mathscr{U}$ is a countably incomplete good ultrafilter on a set of cardinality $\aleph$, then $\mathcal{G}_{\mathscr{U}}$ is an $\left(1, \aleph^{+}\right)$-injective Banach space of density $2^{\aleph}$ by Theorem 6.2. The assertion in (a) now follows from [3].

(b) The space of Gurariu is isomorphic to the space $A(P)$ of continuous affine functions on the Poulsen simplex as proved by Lusky [29]. See also [15] and [28]. Hence $\mathcal{G}_{\mathscr{U}}$ is isomorphic to $A(P)_{\mathscr{U}}$, in turn isometric to the space of continuous affine functions on certain simplex $S$, by Proposition 2.1 in [20]. Thus, the unit ball of $A(S)=A(P)_{\mathscr{U}}$ has extreme points: $1_{S}$ is one. However, $A(S)$, being isomorphic to $\mathcal{G}_{\mathscr{U}}$ cannot be complemented in an $\mathcal{M}$-space. As before, the density character of $A(S)$ equals $2^{\aleph}$ and $A(S)$ is $\left(1, \aleph^{+}\right)$-injective.

The preceding examples are as bad as the generalized continuum hypothesis allows. Indeed, if a Banach space is $\left(1, \aleph^{+}\right)$-injective and has density character $\aleph$, then it is 1-injective and then isometric to a $C(K)$-space; see for instance Corollary 1 in [31]. The presence of an extreme point in part (b) is reminiscent from the early studies on injectivity (cf. [30], [23], [2]).

\section{Projectiveness properties of compact spaces}

The compact spaces arising in Theorem 5.2 constitute a well known class ([6], [7], and [37]) we consider now.

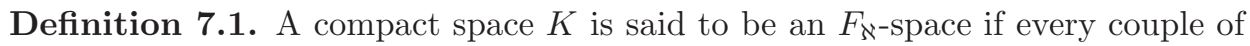
disjoint open subsets of $K$ which are the union of less than $\aleph$ many closed sets have disjoint closures.

The $F_{\aleph_{1}}$-spaces are called simply $F$-spaces. Regarding Theorem 5.2 let us mention that a topological space is called $\left(Q_{\aleph}\right)$-space [2] if every couple of disjoint open subsets of $K$ which are the union of less than $\aleph$ many closures of open sets have disjoint closures. This property is formally weaker than condition (d) in Theorem 5.2, and stronger than (e), because every cozero set is the union of countably many closures of open sets, $f^{-1}(\mathbb{R} \backslash\{0\})=\bigcup \overline{f^{-1}(\mathbb{R} \backslash[-1 / n, 1 / n])}$, so it is actually equivalent to both of them in the case of compact spaces.

The following proposition generalizes a result of Bankston (Theorem 2.3.7 (ii) in [6]) and solves Question 2.3.8 in [6] by showing that the extra condition of being Boolean is not necessary. Its proof is immediate after Theorems 5.2 and 6.2.

Proposition 7.2. Every topological ultracoproduct via a countably incomplete,

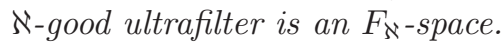

As we mentioned before, a Banach space is 1-injective if and only if it is isometrically isomorphic to $C(K)$ for some extremely disconnected compact space $K$ (see Theorem 2.1 in [39]) and such compacta are precisely the projective elements in the category of compacta and continuous maps, a classical result by Gleason [16] that can be seen in Theorem 10.51 of [38]. Which means that if $\sigma: L \rightarrow M$ is a 
continuous surjection then any continuous map $\varphi: K \rightarrow M$ lifts to $L$ in the sense that there is $\tilde{\varphi}: K \rightarrow L$ such that $\varphi=\sigma \circ \tilde{\varphi}$. Of course this can be rephrased by saying that $C(K)$ is injective in the category of commutative $\mathrm{C}^{*}$-algebras. One may wonder if some natural relativization of this result holds, meaning whether the fact that the space $C(K)$ is injective with respect to a subcategory of Banach spaces is reflected dually by $K$ being projective with respect to some subcategory of compact spaces. If $\mathscr{C}$ is some class of continuous surjections between compact spaces, we say that a compact space $K$ is projective with respect to $\mathscr{C}$ if for every continuous surjection $\pi: L \rightarrow M$ that belongs to $\mathscr{C}$ and every continuous map $f: K \rightarrow M$ there exists a continuous function $g: K \rightarrow M$ such that $\pi g=f$. The first guess would be that $C(K)$ being injective with respect to Banach spaces of density less than $\aleph$ should be equivalent to the Banach space $K$ being projective with respect to compact spaces of weight less than $\aleph$. There is however a serious obstruction for this approach: if $\pi$ is any surjection from the Cantor set $\Delta$ onto the unit interval $\mathbb{I}$ and $K$ is any connected $F$-space, then the only liftable maps $f: K \rightarrow \mathbb{I}$ are the constant ones (Proposition 7.2 provides a good number of such spaces: it is not difficult to realize that ultracoproducts preserve connectedness since a compact space $K$ is connected if and only if the only idempotents in $C(K)$ are 0 and 1 ). There are two ways of avoiding this problem. The first way is to assume $K$ to be totally disconnected or, which is the same, zero-dimensional (Theorem 7.4). The other way is to reduce the subcategory we are dealing with and to consider only compact convex sets and affine maps between them (Theorem 7.10). Before going further let us remark:

Lemma 7.3. Let $\aleph$ be a cardinal number, and $K$ a compact space. The following are equivalent:

(1) Every open cover of every subspace of $K$ has a subcover of cardinality less than $\aleph$.

(2) Every open subset of $K$ is the union of less than $\aleph$ many closed subsets of $K$.

Proof. Suppose (1) holds and let $U$ be an open subset of $K$. Simply consider an open cover of $U$ by open sets $V$ with $\bar{V} \subset U$. Conversely, assume (2) and let $S \subset K$ and $\left\{U_{i}: i \in I\right\}$ a cover of $S$ by open subsets of $K$. Consider $U=\bigcup_{i \in I} U_{i}$. By (2), $U$ is the union of less than $\aleph$ many compact sets, so it is enough to take a finite subcover of each.

We denote by $H L_{\aleph}$ the class of compact spaces satisfying the conditions of the preceding lemma. Observe that this class is stable under continuous images and that it contains all compact spaces of weight less than $\aleph$. A compact space belongs to $H L_{\aleph_{1}}$ if and only if it is hereditarily Lindelöf, if and only if it is perfectly normal. An example of a hereditarily Lindelöf space of uncountable weight is the double arrow space: the lexicographical product of ordered sets $[0,1] \times\{0,1\}$ endowed with the order topology. The equivalence of (1), (2) and (3) in the next result is due to Neville and Lloyd [32]. The fourth condition states that $F_{\aleph}$ spaces are projective with respect to a larger class of spaces than those of weight less than $\aleph$. 
Theorem 7.4. For a compact space $K$ the following are equivalent:

(1) $K$ is a zero-dimensional $F_{\aleph}$-space.

(2) $K$ is projective with respect to surjections $\pi: L \rightarrow M$ such that $w(L)<\aleph$.

(3) $K$ is projective with respect to surjections $\pi: L \rightarrow M$ such that $w(M)<\aleph$ and $w(L) \leq \aleph$.

(4) $K$ is projective with respect to surjections $\pi: L \rightarrow M$ with $L \in H L_{\aleph}$.

Proof. Notice that the implications $(4) \Rightarrow(2)$ and $(3) \Rightarrow(2)$ are trivial. We prove first that (2) implies (1). In order to show that $K$ is a zero-dimensional $F_{\aleph}$-space we shall show that for any disjoint open subsets $A$ and $B$, which are the union of $\kappa<\aleph$ many closed subsets of $K$ there exists a clopen set $C$ such that $A \subset C$ and $B \cap C=\varnothing$. Suppose $A=\bigcup_{\alpha<\kappa} C_{\alpha}$ and $B=\bigcup_{\alpha<\kappa} D_{\alpha}$ where each $C_{\alpha}$ and each $D_{\alpha}$ are closed sets. For every $\alpha<\kappa$ let $f_{\alpha}: K \longrightarrow[-1,1]$ be a continuous function such that

- $\left.f_{\alpha}\right|_{C_{\alpha}}=-1$,

- $\left.f_{\alpha}\right|_{A} \leq 0$,

- $\left.f_{\alpha}\right|_{K \backslash(A \cup B)}=0$,

- $\left.f_{\alpha}\right|_{B} \geq 0$, and

- $\left.f_{\alpha}\right|_{D_{\alpha}}=1$.

Consider the map $f: K \longrightarrow[-1,1]^{\kappa}$ given by $f(x)=\left(f_{\alpha}(x)\right)_{\alpha<\kappa}$. Let also $L=[0,1]^{\kappa} \times\{-1,1\}, \pi: L \longrightarrow[-1,1]^{\kappa}$ be given by $\pi(x, t)=\left(t \cdot x_{\alpha}\right)_{\alpha<\kappa}$ and $M=\pi(L)$. Notice that the image of $f$ is contained in $M$, hence we are in a position to apply the projectiveness property so that there exists $g: K \rightarrow L$ with $\pi g=f$. But then $g(A) \subset[0,1]^{\kappa} \times\{-1\}$ and $g(B) \subset[0,1]^{\kappa} \times\{1\}$, hence there are disjoint clopen sets which separate $A$ and $B$. Conversely, we prove now that (1) implies (3) and (4). So assume now that $K$ is a zero-dimensional $F_{\aleph}$ space. We assume that we are given an onto map $\pi: L \rightarrow M$ like either in (3) or (4), and $f: K \rightarrow M$, and we will find $g: K \rightarrow L$ with $\pi g=f$.

CAse 1. We suppose that $M \in H L_{\aleph}, L \subset M \times\{0,1\}$ and $\pi: L \rightarrow M$ is the first-coordinate projection. Consider

$$
\begin{aligned}
& A=K \backslash f^{-1}[\pi(L \cap M \times\{1\})], \\
& B=K \backslash f^{-1}[\pi(L \cap M \times\{0\})] .
\end{aligned}
$$

These are two disjoint open subsets of $K$ which are moreover the union of less than $\aleph$ many closed sets, because $M \in H L_{\aleph}$. Therefore, since $K$ is a totally disconnected $F_{\aleph}$ space, there exists a clopen set $C \subset K$ such that $A \subset C$ and $B \subset C=\varnothing$. The desired function $g: K \rightarrow L$ can be defined now as $g(x)=(x, 0)$ if $x \in C$ and $g(x)=(x, 1)$ if $x \notin C$.

CAse 2. We suppose that $L \in H L_{\aleph}, L \subset M \times[0,1]$ and $\pi: L \rightarrow M$ is the first-coordinate projection. Let $q: 2^{\omega} \rightarrow[0,1]$ be a continuous surjection from the Cantor set onto the unit interval. Let $L^{\prime}=\left\{(x, t) \in M \times 2^{\omega}:(x, q(t)) \in L\right\}$ and 
let $\pi^{\prime}: L^{\prime} \rightarrow M$ be the first coordinate projection. We shall find a continuous map $g^{\prime}: K \rightarrow L^{\prime}$ such that $\pi^{\prime} g^{\prime}=f$. From $g^{\prime}$ we easily obtain the desired function $g$ by composing with $q$ in the second coordinate. For every $n<m \leq \omega$ let $p_{n}^{m}$ : $M \times 2^{m} \rightarrow M \times 2^{n}$ be the natural projection which forgets about coordinates $i \geq n$ in $2^{m}$. Let $L_{n}=p_{n}^{\omega}\left(L^{\prime}\right)$. Each $L_{n} \subset L \times 2^{n}$ is a member of $H L_{\aleph}$. Hence, by repeated application of the Case 1 proved above, we can construct inductively continuous maps $g_{n}: K \rightarrow L_{n}$ such that $g_{0}=f$ and $\pi_{n}^{n+1} g_{n+1}=g_{n}$. These functions must be of the form $g_{n}(x)=\left(f(x), \gamma_{0}(x), \ldots, \gamma_{n-1}(x)\right)$ for some continuous functions $\gamma_{i}: K \rightarrow 2, i<\omega$. The function $g^{\prime}: K \rightarrow L^{\prime} \subset M \times 2^{\omega}$ is defined as $g^{\prime}(x)=$ $\left(f(x), \gamma_{0}(x), \gamma_{1}(x), \ldots\right)$.

General CASE. We view $L$ as a closed subset of a cube $L \subset 2^{\Gamma}$, where $\Gamma$ is some cardinal. If we are dealing with condition $(3)$, then $\Gamma=\aleph$. Let $G=$ $\{(x, \pi(x)): x \in L\} \subset[0,1]^{\Gamma} \times M$ be the graph of $\pi$, and let $\pi_{1}: G \rightarrow L$ and $\pi_{2}: G \rightarrow M$ be the two coordinate functions. We shall find a continuous function $h: K \rightarrow G$ such that $\pi_{2} h=f$. From this we immediately get the desired lifting as $g=\pi_{1} h$.

For every $\alpha<\beta \leq \Gamma$ let $p_{\alpha}^{\beta}: 2^{\beta} \times M \rightarrow 2^{\alpha} \times M$ be the natural projection and let $G_{\alpha}=p_{\alpha}^{\Gamma}(G)$. If we assume condition (3) then all spaces $G_{\alpha}$ have weight less than $\aleph$, while if we assume (4), then all these spaces belong to $H L_{\aleph}$ because $G$ is homeomorphic to $L$ and this class is stable under taking continuous images. We construct by transfinite induction continuous functions $h_{\alpha}: K \rightarrow G_{\alpha}$ such that $\pi_{2} h_{\alpha}=f$ and such that they are coherent: $p_{\alpha}^{\beta} h_{\beta}=h_{\alpha}$ for $\alpha<\beta$. In the one immediate successor step of the induction, in order to obtain $h_{\alpha+1}$ from $h_{\alpha}$ we are in a position to apply Case 2 above. In the limit step, the function $h_{\beta}$ is uniquely determined by the functions $h_{\alpha}$ with $\alpha<\beta$, similarly as we did in Case 2 .

Corollary 7.5. The following spaces are "projective" with respect to all continuous surjections between metrizable compacta:

- $\mathbb{N}^{*}$, the growth of the integers in its Stone-Čech compactification.

- Ultracoproducts of families of totally disconnected compacta built over countably incomplete ultrafilters.

Corollary 7.6. Totally disconnected F-spaces are projective with respect to hereditarily Lindelöf compact spaces.

Some particular cases of Corollary 7.6 are proven by Przymusiński [33] to the effect of showing that every hereditarily Lindelöf compact space is a continuous image of $\mathbb{N}^{*}$. Yet his arguments require some extra hypotheses which are unnecessary at the end. In the following corollary, we denote by $R O(X)$ the set of all regular open subsets of $X$, that is, those open sets which are interiors of closed sets.

Corollary 7.7. Let $K$ be a totally disconnected $F_{\aleph}$-space. Then $K$ is projective with respect to surjections $\pi: L \rightarrow M$ in which $w(M)<\aleph$ and $|R O(M)| \leq \aleph$.

Proof. Let $f: K \rightarrow L$ as usual, and let $p: G \rightarrow M$ be the Gleason cover of $M$. We refer to [38] for an explanation about Gleason covers. We just recall the facts that 
we need about it: the space $G$ is an extremely disconnected space (that is, projective with respect to the full category of compact spaces), $w(G)=|R O(M)|$ and, and $p: G \rightarrow M$ is an onto continuous map. Since $w(G) \leq \aleph$ and $w(M)<\aleph$, by Theorem 7.4 there exists $h: K \rightarrow G$ such that $p h=f$. Since $G$ is projective, there exists $u: G \rightarrow L$ such that $\pi u=p$. Take $g=u h$.

Corollary 7.8 (Neville and Lloyd). If $\kappa$ is a cardinal for which $\kappa^{+}=2^{\kappa}$, and $K$ is a totally disconnected compact $F_{\kappa^{+}}$-space, then $K$ is projective with respect to all surjections $\pi: L \rightarrow M$ such that $w(M) \leq \kappa$.

Proof. Apply the preceding Corollary for $\aleph=\kappa^{+}$, and notice that one always has $|R O(M)| \leq 2^{w(M)}$ because every open set is the union of a family of open sets from a basis.

Neville and Lloyd [32] asked whether the assumption that $\kappa^{+}=2^{\kappa}$ can be removed. We point out that the compact space constructed by Dow and Hart [13] we used in Theorem 7 of [4] provides a negative answer to their question.

Theorem 7.9. It is consistent that there exists a zero-dimensional compact $F$ space $K$ which is not projective with respect to surjections $\pi: L \rightarrow M$ with $w(M)=\aleph_{0}$.

Proof. Under the assumption that $\mathfrak{c}=\aleph_{2}$ and that $\wp(\mathbb{N}) /$ fin contains a chain of order type $\omega_{2}$, Dow and Hart (Theorem 5.10 in [13]) construct a zero-dimensional compact $F$-space $K$ which does not map onto $\beta \mathbb{N}$. Let $M=\alpha \mathbb{N}$ be the one-point compactification of the natural numbers, $L=\beta \mathbb{N}$ and $\pi: \beta \mathbb{N} \rightarrow M$ defined as $\pi(n)=n$ for $n \in \mathbb{N}$, and $\pi(x)=\infty$ if $x \in \beta \mathbb{N} \backslash \mathbb{N}$. Let $f: K \rightarrow M$ be a continuous surjection. We claim that any continuous map $g: K \rightarrow L$ with $\pi g=f$ must be onto, hence there is no such $g$. The reason is that for every $n \in \mathbb{N}$, if $x_{n}$ is such that $f\left(x_{n}\right)=n, \pi g\left(x_{n}\right)=n$, hence $g\left(x_{n}\right)=n$. Therefore $\mathbb{N} \subset g(K)$, and since $\mathbb{N}$ is dense in $L$, we conclude that $g$ is onto, as desired.

In the next theorem, by a compact convex set we mean a compact convex set lying inside some locally convex space $E$. Actually, every such set $L$ is affinely homeomorphic to a closed convex subset of a cube $[0,1]^{\Gamma}$, where the size of $\Gamma$ can be as small as the weight of $L$. This is a consequence of the fact that continuous linear functionals on $E$ separate points (see Corollary 3.33 in [14]): one takes takes $\Gamma$ as the set of these functionals and then the correspondence $x \mapsto(f(x))_{f \in \Gamma}$ shows that $L$ is affinely homeomorphic to a compact convex subset of $\mathbb{R}^{\Gamma}$, indeed by compactness to a subset of a product of intervals $\prod_{\Gamma}\left[a_{\gamma}, b_{\gamma}\right]$, which is in turn affinely homeomorphic to $[0,1]^{\Gamma}$.

Theorem 7.10. Suppose $\aleph \geq \aleph_{1}$. For a compact space $K$ the following are equivalent:

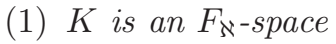

(2) For every continuous affine surjection $\pi: L \rightarrow M$ between compact convex sets with $w(L)<\aleph$, and every continuous function $f: K \rightarrow M$, there exists a continuous function $g: K \rightarrow L$ such that $\pi g=f$. 
(3) As above with $w(M)<\aleph$ and $w(L) \leq \aleph$.

(4) As above with $L \in H L_{\aleph}$.

Proof. It is clear that $(3) \Rightarrow(2)$ and that $(4) \Rightarrow(2)$. We shall prove that (2) implies (1), and that (1) implies (3) and (4). Suppose first that (2) holds, and we shall show that the second condition of Theorem 5.2 holds for any cardinal $\Gamma<\aleph$. Let $f_{\alpha}, g_{\alpha}: K \rightarrow[0,1]$, with $\alpha<\Gamma$, be two families of continuous functions such that $f_{\alpha} \leq g_{\beta}$ for every $\alpha, \beta<\Gamma$. Consider

$$
\begin{aligned}
M & =\left\{\left(\left(t_{\alpha}\right)_{\alpha<\Gamma},\left(s_{\alpha}\right)_{\alpha<\Gamma}\right) \in[0,1]^{\Gamma} \times[0,1]^{\Gamma}: \sup _{\alpha<\Gamma} t_{\alpha} \leq \inf _{\alpha<\Gamma} s_{\alpha}\right\}, \\
L & =\left\{\left(\left(t_{\alpha}\right)_{\alpha<\Gamma}, r,\left(s_{\alpha}\right)_{\alpha<\Gamma}\right) \in[0,1]^{\Gamma} \times[0,1] \times[0,1]^{\Gamma}: \sup _{\alpha<\Gamma} t_{\alpha} \leq r \leq \inf _{\alpha<\Gamma} s_{\alpha}\right\} .
\end{aligned}
$$

Let $\pi: L \rightarrow M$ be the natural surjection which forgets the intermediate coordinate $r$, and let also $f: K \rightarrow M$ be given by

$$
f(x)=\left(f_{\alpha}(x)_{\alpha<\Gamma}, g_{\alpha}(x)_{\alpha<\Gamma}\right) .
$$

We are in a position to apply the statement of part (2), so that there is a function $g: K \rightarrow L$ such that $\pi(g(x))=f(x)$. If we look at the composition of $g$ with the projection on the central coordinate $r$ of $L$, we obtain a continuous function $h: K \rightarrow[0,1]$ such that $f_{\alpha} \leq h \leq g_{\alpha}$ for every $\alpha<\Gamma$. This proves that $K$ is an $F_{\aleph}$-space.

Now we proceed to the proof that (1) implies (3) and (4), so we suppose that $K$ is an $F_{\aleph}$-space, $\pi: L \rightarrow M$ is a continuous affine surjection and $f: K \rightarrow M$ is a continuous surjection. We want to show that, under the hypotheses of either (3) or (4), we get a continuous function $g: K \rightarrow L$ such that $\pi g=f$. We consider $M$ to be a closed convex subset of a cube, $M \subset[0,1]^{\Gamma}$ and we call $\pi_{\alpha}: M \rightarrow[0,1]$ to the projection on the $\alpha$-th coordinate. The first step is to find the desired function $g$ under the following assumption (which can be considered the analogue of considering a Banach superspace of codimension 1):

STEP 1. We assume $M \in H L_{\aleph}$ and there exists a continuous affine function $\phi: L \rightarrow[0,1]$ such that the map $(\pi, \phi): L \rightarrow M \times[0,1]$ given by $(\pi, \phi)(x)=$ $(\pi(x), \phi(x))$ is one-to-one.

In this case, we shall view $L$ as a closed convex subset of $M \times[0,1]$, so that $\pi$ and $\phi$ are just the projections on the first and second coordinate. To find the desired function $g: K \rightarrow L$ is equivalent to find a continuous function $\gamma: K \rightarrow[0,1]$ such that $(f(x), \gamma(x)) \in L$ for every $x \in K$. Let $\left\{q_{n}: n<\omega\right\}$ be a countable dense subset of $[0,1]$. We shall define by induction continuous functions $\gamma_{n}^{-}, \gamma_{n}^{+}: K \rightarrow$ $[0,1]$ such that $\gamma_{n}^{-} \leq \gamma_{m}^{+}$for every $n, m$, and then $\gamma$ will be chosen such that $\gamma_{n}^{-} \leq \gamma \leq \gamma_{m}^{+}$for every $n, m$. For each $n$, define

$$
\begin{aligned}
& U_{n}^{-}=\left\{y \in M:(y, t) \notin L \text { for every } t \in\left[q_{n}, 1\right]\right\} \backslash \pi\left(L \cap\left(M \times\left[q_{n}, 1\right]\right)\right), \\
& U_{n}^{+}=\left\{y \in M:(y, t) \notin L \text { for every } t \in\left[0, q_{n}\right]\right\} \backslash \pi\left(L \cap\left(M \times\left[0, q_{n}\right]\right)\right),
\end{aligned}
$$

which are two disjoint open subsets of $M$. Since $M \in H L_{\aleph}, f^{-1}\left(U_{n}^{-}\right)$and $f^{-1}\left(U_{n}^{+}\right)$ are disjoint open subsets of $K$ which are moreover unions of less than $\aleph$ many closed 


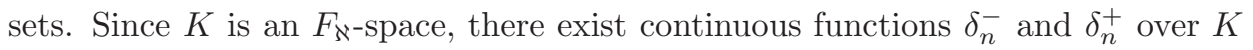
such that

$$
\begin{array}{ll}
0 \leq \delta_{n}^{-} \leq q_{n},\left.\quad \delta_{n}^{-}\right|_{f^{-1}\left(U_{n}^{-}\right)} \equiv 0, & \left.\delta_{n}^{-}\right|_{f^{-1}\left(U_{n}^{+}\right)} \equiv q_{n}, \\
q_{n} \leq \delta_{n}^{+} \leq 1, & \left.\delta_{n}^{+}\right|_{f^{-1}\left(U_{n}^{-}\right)} \equiv q_{n},\left.\quad \delta_{n}^{+}\right|_{f^{-1}\left(U_{n}^{+}\right)} \equiv 1 .
\end{array}
$$

A priori, it may be false that $\delta_{n}^{-} \leq \delta_{m}^{+}$for every $n, m$, so in order to make sure of this we define inductively:

$$
\gamma_{n}^{-}=\min \left\{\delta_{n}^{-}, \gamma_{m}^{+}: m<n\right\}, \quad \gamma_{n}^{+}=\max \left\{\delta_{n}^{+}, \gamma_{m}^{-}: m<n\right\} .
$$

It is easy to see (using the fact that if $q_{i}<q_{j}$, then $f^{-1}\left(U_{i}^{-}\right) \subset f^{-1}\left(U_{j}^{-}\right)$and $\left.f^{-1}\left(U_{i}^{+}\right) \supset f^{-1}\left(U_{j}^{+}\right)\right)$that these new functions still keep the key properties that

$$
\begin{aligned}
& 0 \leq \gamma_{n}^{-} \leq q_{n},\left.\quad \gamma_{n}^{-}\right|_{f^{-1}\left(U_{n}^{-}\right)} \equiv 0,\left.\quad \gamma_{n}^{-}\right|_{f^{-1}\left(U_{n}^{+}\right)} \equiv q_{n}, \\
& q_{n} \leq \gamma_{n}^{+} \leq 1,\left.\quad \gamma_{n}^{+}\right|_{f^{-1}\left(U_{n}^{-}\right)} \equiv q_{n},\left.\quad \gamma_{n}^{+}\right|_{f^{-1}\left(U_{n}^{+}\right)} \equiv 1 \text {. }
\end{aligned}
$$

Since $K$ is in particular an $F$-space, there exists a continuous function $\gamma: K \rightarrow$ $[0,1]$ such that $\gamma_{n}^{-} \leq \gamma \leq \gamma_{n}^{+}$for all $n$. We have to show that $(f(x), \gamma(x)) \in L$ for every $x \in K$. Given $x \in K$, let

$$
I=\{t \in[0,1]:(f(x), t) \in L\}=\phi\left(\pi^{-1}[f(x)]\right) .
$$

Since $\phi$ and $\pi$ are affine, $I=[a, b]$ is a closed interval. In order to check that $\gamma(x) \in I$, we show that $q_{n} \leq \gamma(x) \leq q_{m}$ whenever $q_{n}<a$ and $q_{m}>b$. For example, if $q_{n}<a$, then this means that $f(x) \in U_{n}^{+}, x \in f^{-1}\left(U_{n}^{+}\right)$, so $q_{n}=\gamma_{n}^{-}(x) \leq \gamma(x)$. Analogously, if $q_{m}>b$, then $x \in f^{-1}\left(U_{m}^{-}\right)$, and $\gamma(x) \leq \gamma_{m}^{+}(x)=q_{m}$. This finishes the proof under the assumption made in Step 1.

General CASE. We view now $L$ as compact convex set of the Hilbert cube $[0,1]^{\Gamma}$ (with $\Gamma=\aleph$ when we are under the assumptions of case $(3)$ ) and we call $\chi_{\alpha}: L \rightarrow[0,1]$ the coordinate functions, $\alpha<\Gamma$. For every $\alpha$, we consider the map $h_{\alpha}: L \rightarrow M \times[0,1]^{\alpha}$ given by $h_{\alpha}(z)=\left(\pi(z), \chi_{\beta}(z)_{\beta<\alpha}\right)$, and we call $L_{\alpha}=$ $h_{\alpha}(L) \subset M \times[0,1]^{\alpha}$ the image of this continuous function. For $\alpha<\beta$, we also call $p_{\alpha}^{\beta}: L_{\beta} \rightarrow L_{\alpha}$ the continuous surjection which forgets about coordinates $t_{i}$ with $i \geq \alpha$. We construct by transfinite induction a sequence of coherent liftings $g_{\alpha}: K \rightarrow L_{\alpha}, \alpha<\Gamma$, that is, functions satisfying $g_{0}=f$ and $p_{\alpha}^{\beta} g_{\beta}=g_{\alpha}$ whenever $\alpha<\beta$. Notice that this is actually equivalent to finding continuous functions $\gamma_{\alpha}: K \rightarrow[0,1]$ such that $g_{\alpha}(x)=\left(f(x), \gamma_{\beta}(x)_{\beta<\alpha}\right) \in L_{\alpha}$ for every $x \in K$ and $\alpha \leq \Gamma$. In the inductive process $g_{\alpha+1}$ is obtained from $g_{\alpha}$ by applying Step 1, while in the limit ordinals one has to take $g_{\beta}(x)=\left(f(x), \gamma_{\alpha}(x)_{\alpha<\beta}\right)$. Notice that Step 1 can be applied because $L_{\alpha} \in H L_{\aleph}$ : if we are in case $(3)$, we took $\Gamma=\aleph$, so $w\left(L_{\alpha}\right)<\aleph$, while in case (4) $L_{\alpha}$ is a continuous image of $L$ and $L \in H L_{\aleph}$. Let $g_{\Gamma}: K \rightarrow M \times L$ be the final output of this inductive construction. We have that $p_{0}^{\Gamma} g_{\Gamma}=f$. Let $g: K \rightarrow L$ be obtained by projecting $g_{\Gamma}$ on the second coordinate, so that we can write $g^{\Gamma}(x)=(f(x), g(x))$. The fact that $g^{\Gamma}(x) \in L_{\Gamma}$ implies that $\pi(g(x))=f(x)$, so $g$ is the map that we were looking for. 


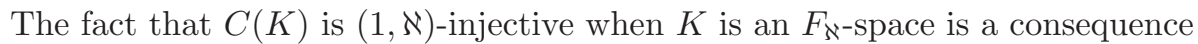
of Theorem 7.10. Suppose we have $Y \subset X$ Banach spaces with dens $X<\aleph$ and $t: Y \rightarrow C(K)$ an operator of norm 1. We can apply part (2) of Theorem 7.10 to $\pi: B_{X^{*}} \rightarrow B_{Y^{*}}$ and the mapping $f: K \rightarrow B_{Y^{*}}$ given by $f(x)=t^{*}\left(\delta_{x}\right)$. We obtain a weak*-continuous function $g: K \rightarrow B_{X^{*}}$ such that $\pi g=f$. Then, the formula $T(x)(k)=\|x\| g(k)(x /\|x\|), x \in X, k \in K$, defines an extension of $t$ of norm 1 .

\section{Open problems}

(1) Find homological characterizations of $\aleph$-injectivity and universal $\aleph$-injectivity in ZFC. Proposition 3.2 characterizes $\left(2^{\aleph}\right)^{+}$-injectivity; in particular, it characterizes $\aleph_{2}$-injectivity under $\mathrm{CH}$ (a Banach space $E$ is $\aleph_{2}$-injective if and only if it is complemented in every superspace $W$ such that $W / E$ is a quotient of $\ell_{\infty}$ ) and $\aleph^{+}$-injectivity under GCH (a Banach space $E$ is $\aleph^{+}$-injective if and only if it is complemented in every superspace $W$ such that $W / E$ is a quotient of $\left.\ell_{\infty}(\aleph)\right)$.

(2) Find a characterization of universal $(1, \aleph)$-injectivity by means of intersection of families of balls.

(3) Is universal $\aleph$-injectivity a 3 -space property?

(4) Is it consistent that $C\left(\mathbb{N}^{*}\right)$ is $\aleph_{2}$-injective? Recall that we have already shown that $C\left(\mathbb{N}^{*}\right)$ is not $\left(1, \aleph_{2}\right)$-injective nor $\mathfrak{c}^{+}$-injective.

(5) Are Lindenstrauss ultraproducts via countably incomplete $\aleph$-good ultrafilters universally $\aleph$-injective spaces in ZFC? (They are universally $(1, \aleph)$-injective under GCH.)

(6) Prove or disprove that every ultraproduct built over a countably incomplete, $\aleph$-good ultrafilter is $\aleph$-injective as long as it is a $\mathscr{L}_{\infty}$-space.

\section{References}

[1] Albiac, F. And Kalton, N. J.: Topics in Banach space theory. Graduate Texts in Mathematics 233, Springer, New York, 2006.

[2] Aronszajn, N. and Panitchpakdi, P.: Extension of uniformly continuous transformations and hyperconvex metric spaces. Pacific J. Math. 6 (1956), 405-439.

[3] Avilés, A., Cabello, F., Castillo, J. M. F., González, M. and Moreno, Y.: Banach spaces of universal disposition. J. Funct. Anal. 261 (2011), no. 9, 2347-2361.

[4] Avilés, A., Cabello, F., Castillo, J. M.F., González, M. and Moreno, Y.: On separably injective Banach spaces. Adv. Math. 234 (2013), 192-216.

[5] Avilés, A., Cabello, F., Castillo, J. M. F., González, M. and Moreno, Y.: On ultrapowers of Banach spaces of type $\mathscr{L}_{\infty}$. Fundamenta Math. 222 (2013), no. 3, $195-212$.

[6] Bankston, P.: Reduced coproducts of compact Hausdorff spaces. J. Symbolic Logic 52 (1987), no. 2, 404-424.

[7] Bankston, P.: A survey of ultraproduct constructions in general topology. Topology Atlas 8 (1993), 1-32. 
[8] Cabello Sánchez, F. and Castillo, J. M. F.: The long homology sequence for quasi-Banach spaces, with applications. Positivity 8 (2004), no. 4, 379-394.

[9] Castillo, J. M.F. And GonzÁlez, M.: Three-space problems in Banach space theory. Lecture Notes in Mathematics 1667, Springer-Verlag, Berlin, 1997.

[10] Castillo, J. M. F. And Plichko, A.: Banach spaces in various positions. J. Funct. Anal. 259 (2010), no. 8, 2098-2138.

[11] Chang, C. C. And Keisler, H. J.: Model theory. Studies in Logic and the Foundations of Mathematics 73, North-Holland, Amsterdam, 1990.

[12] Comfort, W.W. And Negrepontis, S.: The theory of ultrafilters. Die Grundlehren der Matematischen Wissenchaften 211, Springer-Verlag, New YorkHeidelberg, 1974.

[13] Dow, A. And HART, K.P.: Applications of another characterization of $\beta \mathbb{N} \backslash \mathbb{N}$. Topology Appl. 122 (2002), no. 1-2, 105-133.

[14] Fabian, M., Habala, P., Hájek, P., Montesinos, V. and Zizler, V.: Banach space theory. The basis for linear and nonlinear analysis. CMS Books in Mathematics/Ouvrages de Mathématiques de la SMC, Springer, New York, 2011.

[15] Fonf, V. P., Lindenstrauss, J. and Phelps, R. R.: Infinite dimensional convexity. In Handbook of the geometry of Banach spaces, Vol. I, 599-670. North-Holland, Amsterdam, 2001.

[16] Gleason, A. M.: Projective topological spaces. Illinois J. Math. 2 (1958), 482-489.

[17] Hasanov, V.S.: Some universally complemented subspaces of $m(\Gamma)$. Mat. Zametki 27 (1980), no. 1, 105-108, 159.

[18] Hausdorff, F.: Summen von $\aleph_{1}$ Mengen. Fund. Math. 26 (1936), 241-255.

[19] Heinrich, S.: Ultraproducts in Banach space theory. J. Reine Angew. Math. 313 (1980), 72-104.

[20] Heinrich, S.: Ultraproducts of $L_{1}$-predual spaces. Fund. Math. 113 (1981), no. 3, $221-234$.

[21] Henriksen, M.: Some remarks on a paper of Aronszajn and Panitchpakdi. Pacific J. Math. 7 (1957), 1619-1621.

[22] KeIsLeR, H. J.: The ultraproduct construction. In Ultrafilters across mathematics, 163-179. Contemporary Mathematics 530, Amer. Math. Soc., Providence, RI, 2010.

[23] Kelley, J. L.: Banach spaces with the extension property. Trans. Amer. Math. Soc. 72 (1952), 323-326.

[24] Lindenstrauss, J.: Extension of compact operators. Mem. Amer. Math. Soc. 48 (1964), 1-112.

[25] Lindenstrauss, J.: On the extension of operators with range in a $C(K)$ space. Proc. Amer. Math. Soc. 15 (1964), 218-225.

[26] Lindenstrauss, J.: Automorphisms in $c_{0}, l_{1}$ and $m$. Israel J. Math. 7 (1969), $227-239$.

[27] Lindenstrauss, J. And Tzafriri, L.: Classical Banach spaces. I. Sequence spaces. Ergebnisse der Mathematik und ihrer Grenzgebiete 92, Springer-Verlag, Berlin-New York, 1977.

[28] Lusky, W.: Separable Lindenstrauss spaces. In Functional analysis: surveys and recent results (Proc. Conf., Paderhorn, 1976), 15-28. Notas Mat. 63, North-Holland, Amsterdam-New York, 1977. 
[29] Lusky, W.: A note on Banach spaces containing $c_{0}$ or $C_{\infty}$. J. Funct. Anal. 62 (1985), no. 1, 1-7.

[30] Nachbin, L.: A theorem of the Hahn-Banach type for linear transformations. Trans. Amer. Math. Soc. 68 (1950), 28-46.

[31] Neville, C. W.: Banach spaces with a restricted Hahn-Banach extension property. Pacific J. Math. 63 (1976), no. 1, 201-212.

[32] Neville, C. W. And Lloyd, S. P.: ふ-projective spaces. Illinois J. Math. 25 (1981), no. $1,159-168$

[33] Przymusiński, T. C.: Perfectly normal compact spaces are continuous images of $\beta \mathbb{N} \backslash \mathbb{N}$. Proc. Amer. Math. Soc. 86 (1982), no. 3, 541-544.

[34] Rosenthal, H. P.: On relatively disjoint families of measures, with some applications to Banach space theory. Studia Math. 37 (1970), 13-36.

[35] Seever, G. L.: Measures on F-spaces. Trans. Amer. Math. Soc. 133 (1968), 267280.

[36] Sims, B.: "Ultra"-techniques in Banach space theory. Queen's Papers in Pure and Applied Mathematics 60, Queen's University, Kingston, ON, 1982.

[37] Swardson, M. A.: A generalization of $F$-spaces and some topological characterizations of GCH. Trans. Amer. Math. Soc. 279 (1983), no. 2, 661-675.

[38] Walker, R. C.: The Stone-Čech compactification. Ergebnisse der Mathematik und ihrer Grenzgebiete 83, Springer-Verlag, New York-Berlin, 1974.

[39] Zippin, M.: Extension of bounded linear operators. In Handbook of the geometry of Banach spaces, Vol. 2, 1703-1741. North-Holland, Amsterdam, 2003.

Received May 5, 2013; revised February 1, 2014.

Antonio AvilÉs: Departamento de Matemáticas, Universidad de Murcia, 30100 Espinardo, Murcia, Spain.

E-mail: avileslo@um.es

FÉlix Cabello Sánchez: Departamento de Matemáticas, Universidad de Extremadura, Avenida de Elvas s/n, 06011 Badajoz, Spain.

E-mail: fcabello@unex.es

Jesús M.F. Castillo: Departamento de Matemáticas, Universidad de Extremadura, Avenida de Elvas s/n, 06011 Badajoz, Spain.

E-mail: castillo@unex.es

Manuel GonzÁlez: Departamento de Matemáticas, Universidad de Cantabria, Avenida los Castros s/n, 39071 Santander, Spain.

E-mail: manuel.gonzalez@unican.es

Yolanda Moreno: Escuela Politécnica, Universidad de Extremadura, Avenida de la Universidad s/n, 10071 Cáceres, Spain.

E-mail: ymoreno@unex.es

The research of the first author has been supported in part by project MTM2014-54182. The research of the last four authors has been supported in part by project MTM2013-45643. 